\title{
Exploring Various Flux Vector Splittings for the Magnetohydrodynamic System
}

\author{
By \\ Dinshaw S. Balsara ${ }^{1}$ Gino Montecinos ${ }^{2}$ and Eleuterio F. Toro ${ }^{3}$ \\ ${ }^{1}$ Physics and ACMS Departments, University of Notre Dame, Notre Dame, Indiana, USA \\ (dbalsara@nd.edu) \\ ${ }^{2}$ Center for Mathematical Modeling, University of Chile, Chile (gmontecinos@ dim.uchile.cl) \\ ${ }^{3}$ Laboratory of Applied Mathematics, DICAM, University of Trento, Trento, Italy \\ (eleuterio.toro@unitn.it)
}

\begin{abstract}
In this paper we explore flux vector splittings for the MHD system of equations. Our approach follows the strategy that was initially put forward in (E.F. Toro and Vázquez-Cendón, Flux splitting schemes for the Euler equations, Computers \& Fluids, 70 (2012) 1-12). We split the flux vector into an advected sub-system and a pressure sub-system. The eigenvalues and eigenvectors of the split sub-systems are then studied for physical suitability. Not all flux vector splittings for MHD yield physically meaningful results. We find one that is completely useless, another that is only marginally useful and one that should work well in all regimes where the MHD equations are used. Unfortunately, this successful flux vector splitting turns out to be different from the Zha-Bilgen flux vector splitting. The eigenvalues and eigenvectors of this favorable FVS are explored in great detail in this paper.

The pressure sub-system holds the key to finding a successful flux vector splitting. The eigenstructure of the successful flux vector splitting for MHD is thoroughly explored and orthonormalized left and right eigenvectors are explicitly catalogued. We present a novel approache to the solution of the Riemann problem formed by the pressure sub-system for the
\end{abstract}


MHD equations. Once the pressure sub-system is solved, the advection sub-system follows naturally. Our method also works very well for the Euler system.

Our FVS successfully captures isolated, stationary contact discontinuities in MHD. However, we explain why any FVS for MHD is not adept at capturing isolated, stationary Alfvenic discontinuities. Several stringent one-dimensional Riemann problems are presented to show that the method works successfully and can effectively capture the full panoply of wave structures that arise in MHD. This includes compound waves and switch-on and switch-off shocks that arise because of the non-convex nature of the MHD system.

\section{I) Introduction}

The magnetohydrodynamic (MHD) equations describe the large-scale dynamics of a plasma that is threaded by magnetic fields. Because such magnetized plasmas occur frequently in nature, the MHD equations play an important role in various areas of astrophysics, space physics and fusion research. Due to their importance, there is a great deal of interest in their numerical solution. Like the Euler system, the MHD equations form a hyperbolic system. Unlike the Euler system, the MHD equations give rise to a richer foliation of waves. While the Euler system yields a three-fold foliation of waves, the MHD equations give rise to a seven-fold foliation of waves. Thus a characteristic analysis of the MHD equations in one dimension reveals the presence of right- and left-going fast and slow magnetosonic waves, right- and left-going Alfven waves and an entropy wave is centrally located relative to these right- and left-going wave families. The eigenstructure of the MHD equations and the corresponding shock theory has been studied by Jefferey and Taniuti [35]. The eigenstructure for the Euler system is easily analyzed; the eigenstructure for the MHD system is considerably more complicated and fraught with degeneracies that have to be resolved (Brio and $\mathrm{Wu}$ [18], Roe and Balsara [48]). The Euler system has a convex flux, whereas the MHD system has a non-convex flux. The non-convexity of the flux enables the MHD system to realize wave structures that don't find analogues in hydrodynamics. Thus the MHD system can support compound waves and switch-on and switchoff shocks and rarefactions. An analysis of the full panoply of wave structures plays a pivotal role in the design of numerical schemes for the solution of these hyperbolic PDE systems. A good numerical scheme should be able to reproduce the full range of wave structures that the MHD system supports. While several different types of numerical solution techniques have been 
invented over the years, attention still gravitates towards the higher order Godunov schemes which are built on Riemann solver technology. The goal of this paper is to explore flux vector splitting-based Riemann solvers for the MHD system.

The fluxes for the Euler equations are homogeneous in terms of their conserved variables. By contrast, the MHD fluxes are non-homogeneous in terms of their conserved variables, which impedes the easy construction of Riemann solvers. While exact Riemann solvers have been constructed for Euler flow (Godunov [30], van Leer [58], Colella [21]), it has proved difficult to obtain serviceable exact Riemann solvers for the MHD system that work efficiently in numerical codes (Falle [28]). Even so, linearized Riemann solvers for the Euler system (Roe [46], Harten [33]) have been extended to the MHD system (Brio and Wu [18], Cargo and Gallice [19], Balsara [2]). Likewise, the highly popular HLL and HLLC Riemann solvers for the Euler system (Harten et al. [34], Toro et al. [53], [54], Chakraborty \& Toro [20], Batten et al. [16]) have seen extension to MHD (Gurski [31], Li [36], Miyoshi and Kusano [42]). See also, Billett \& Toro [17] for WAF schemes for Euler flow. The HLLEM Riemann solver for Euler flow (Einfeldt [26], Einfeldt et al. [27]) has also been recently improved dramatically and extended to MHD and other systems (Dumbser and Balsara [24]). Modern HLLEM schemes are based on self-similar formulations that were first developed in the context of multidimensional Riemann solvers in Balsara [10]. The local Lax-Friedrichs (LLF) Riemann solver (Rusanov [49]) has also seen frequent use. The LLF flux is also referred to as the Rusanov flux. Osher and Solomon [44] and Dumbser \& Toro [23] presented approximate Riemann solvers based on path integral methods in phase space and they are also known to work well for MHD. The MHD system also has to satisfy a divergence-free constraint for the magnetic field and that has led to the invention of novel reconstruction techniques (Balsara [3], [4], [5], Balsara and Dumbser [13], Xu et al. [62]) as well as multidimensional Riemann solvers (Balsara [6], [7], [10], [12], Balsara, Dumbser and Abgrall [9], Balsara and Dumbser [11], Balsara et al. [14], Vides et al. [59]).

The Riemann solvers described in the previous paragraph all treated the flux as a single entity. There is another philosophy for arriving at a Riemann solver. It consists of viewing the numerical flux as being made up of different parts. This goes under the name of flux vector splitting (FVS). Thus one part of the flux might represent flow entities that are predominantly advected while another part of the flux might predominantly respond to variations in the 
pressure. One rather popular FVS-based Riemann solver consists of the Advection Upstream Splitting Method (AUSM) method (Liou et al. [41], Liou and Steffen [40], Liou [37], [38], [39]). Another analogous FVS was presented by Zha and Bilgen [63]. The common perception is that FVS schemes are easier because they do not require a detailed implementation of the eigenstructure in a numerical code. With ingenious adjustment of the limiting strategies they can even be made to work well on a large range of test problems. Even so, this need to adjust the limiting strategies, and consequently the dissipation, might seem dissatisfying to some practitioners. Toro and Vázquez [55] were able to analyze flux vector splittings for the Euler system including the AUSM and Zha-Bilgen Riemann solvers. Their analysis revealed several deficiencies in existing FVS strategies. Toro and Vázquez [55] then went on to find a particularly favorable FVS of their own for the Euler system. The Toro and Vázquez [55] framework for analyzing flux vector splittings for hyperbolic conservation laws represents a radical departure from the pre-existing style of designing flux vector splittings. However, Toro and Vázquez were able to show that their approach yields rich dividends. The goal of this paper is to extend the Toro and Vázquez philosophy for flux vector splitting to numerical MHD. We mention for the sake of completeness that FVS strategies for MHD based on the Liou and Steffen or Zha-Bilgen philosophies have indeed been attempted (Agarwal et al. [1], Han et al. [32], Xisto et al. [61] and Shen et al. [51]).

In this paper we explore various flux vector splittings for the MHD system of equations. This exploration is carried out within the context of the Toro and Vázquez [55] framework for analyzing flux vector splittings for hyperbolic conservation laws. Their strategy consists of writing the Euler flux as an advected sub-system and a pressure sub-system. The method has drawn considerable recent interest and extensions (Toro et al. [56], Tiam-Kapen and Ghislain [57], Xie et al. [60]). The Toro-Vázquez FVS is differentiated from the other previous FVS approaches in that it treats each of those sub-systems as a hyperbolic system whose wave propagation properties have to be studied and understood. Based on their deep analysis, Toro and Vázquez find that the popular Liou and Steffen AUSM Riemann solver has serious deficiencies. The Zha-Bilgen FVS is also a poor performer for reasons that become apparent via their analysis. While Toro and Vázquez restrict attention to Euler flow, we take their methods further to include a study of MHD flows. We find that some flux vector splittings for the MHD system are entirely unsuitable; others have a limited but viable range of applicability. We also find one FVS that is 
applicable over the entire range of conditions for which the MHD system is likely to be used in practical computations. It is also worth mentioning that the Toro-Vázquez FVS has been pivotal in achieving semi-implicit schemes for Euler flow (Dumbser and Casulli [25]). It is, therefore, hoped that the present analysis of flux vector splittings for MHD will play a similar role in the design of semi-implicit schemes for MHD flow.

Once a favorable FVS strategy is found for the MHD equations, we study the resulting pressure sub-system in considerable detail. This is because the pressure sub-system holds the key to understand the propagation of the advection sub-system. The eigenstructure of the favorable pressure sub-system that we find here is thoroughly analyzed. A simple but very effective HLLEM type Riemann solver is constructed for the evaluation of the numerical flux that arises from the pressure sub-system. An alternative linearization that was originally suggested by Toro and Vázquez is also found to work well for the pressure sub-system. Once the pressure subsystem is solved for, it yields the correct advection velocity with which the advection sub-system can be evolved. The result is a numerical flux for MHD that is made up of advected and pressure parts.

Our FVS successfully captures isolated, stationary contact discontinuities in MHD. However, we explain why any FVS for MHD is not adept at capturing isolated, stationary Alfvenic discontinuities. Several stringent one-dimensional Riemann problems are presented to show that the method works successfully and can effectively capture the full panoply of wave structures that arise in MHD. This includes compound waves and switch-on and switch-off shocks that arise because of the non-convex nature of the MHD system.

The present paper demonstrates the good performance of our FVS for time-explicit MHD simulations. Other very efficient Riemann solvers for MHD have recently been documented in the literature (Dumbser and Balsara [24]). These newer Riemann solvers are based on flux difference splitting and can capture isolated, stationary contact discontinuities as well as isolated, stationary Alfven waves exactly. By contrast, the use of a flux vector splitting in this work restricts the Riemann solver presented here in the following sense. Our present Riemann solver can only capture isolated, stationary contact discontinuities; but it cannot capture isolated, stationary Alfven waves exactly. The FVS-based Riemann solver presented here and the HLLEM Riemann solver from (Dumbser and Balsara [24]) have comparable computational 
complexity because both Riemann solvers draw on the same HLLEM philosophy. So, it may seem that the present FVS-based Riemann solver offers no particular advantage for time-explicit methods. However, we do point out that semi-implicit methods are strongly dependent on obtaining a good flux vector splitting. Please see Park and Munz [45] or Noelle et al. [43] for examples. For such situations, we expect the FVS approach to show significant advantages. The present study is undertaken with an eye towards such semi-implicit applications. In order to build a good semi-implicit scheme for all-speed MHD flows, one has to first verify that the flux vector splitting works well for time-explicit MHD flows. Such a verification is provided in this paper.

In Section II we follow the philosophy of Toro and Vázquez to analyze various possible flux vector splittings for MHD and catalogue their strengths and weaknesses. We identify one FVS for the advection and pressure sub-systems that would be successful in all possible regimes where the MHD equations are likely to be used. In Section III we build an HLLEM type Riemann solver for the pressure sub-system and relate it to a full Riemann solver for MHD that is based on a flux vector splitting. Section IV presents several one-dimensional MHD Riemann problems as tests of our new flux vector splitting. Section V presents some conclusions.

\section{II) The MHD System and an Exploration of Various Flux Vector Splittings}

Let us start with the one-dimensional MHD system written as:-

$$
\frac{\partial}{\partial t}\left(\begin{array}{c}
\rho \\
\rho \mathrm{v}_{x} \\
\rho \mathrm{v}_{y} \\
\rho \mathrm{v}_{z} \\
\varepsilon \\
\mathrm{B}_{x} \\
\mathrm{~B}_{y} \\
\mathrm{~B}_{z}
\end{array}\right)+\frac{\partial}{\partial x}\left(\begin{array}{c}
\rho \mathrm{v}_{x} \\
\rho \mathrm{v}_{x}^{2}+\mathrm{P}+\mathrm{B}^{2} / 8 \pi-\mathrm{B}_{x}^{2} / 4 \pi \\
\rho \mathrm{v}_{x} \mathrm{v}_{y}-\mathrm{B}_{x} \mathrm{~B}_{y} / 4 \pi \\
\rho \mathrm{v}_{x} \mathrm{v}_{z}-\mathrm{B}_{x} \mathrm{~B}_{z} / 4 \pi \\
\left(\varepsilon+\mathrm{P}+\mathrm{B}^{2} / 8 \pi\right) \mathrm{v}_{x}-\mathrm{B}_{x}(\mathbf{v} \cdot \mathbf{B}) / 4 \pi \\
0 \\
\left(\mathrm{v}_{x} \mathrm{~B}_{y}-\mathrm{v}_{y} \mathrm{~B}_{x}\right) \\
-\left(\mathrm{v}_{z} \mathrm{~B}_{x}-\mathrm{v}_{x} \mathrm{~B}_{z}\right)
\end{array}\right)=0
$$

Here $\mathbf{v}=\mathrm{v}_{x} \hat{x}+\mathrm{v}_{y} \hat{y}+\mathrm{v}_{z} \hat{z}$ is the velocity vector; $\mathbf{B}=\mathrm{B}_{x} \hat{x}+\mathrm{B}_{y} \hat{y}+\mathrm{B}_{z} \hat{z}$ is the magnetic field vector; $\rho$ is the density; $\mathrm{P}$ is the pressure; $\varepsilon=\mathrm{P} /(\gamma-1)+\rho \mathbf{v}^{2} / 2+\mathbf{B}^{2} / 8 \pi$ is the energy density and $\gamma$ is the polytropic index. For physical realizability, we have to have $\gamma \geq 1$. 
The purpose of these FVS schemes is to split the equations into a predominantly advective part and another part that predominantly carries the pressure fluctuations. This results in two fluxes, an advective flux (which is written in the form of an advective velocity times a vector of quantities that are to be advected) and a pressure flux (which carries the pressure fluctuations). The resulting flux vector splitting yields two sub-systems whose wave-propagation characteristics can be analyzed in keeping with the philosophy of Toro and Vázquez. In the process of making a FVS one does change the wave propagation characteristics of the two resulting sub-systems. The goal, however, is to retain certain desirable wave propagation characteristics that are closest to the physics. In the Toro-Vázquez philosophy, it may even be desirable to have a pressure sub-system for which the fluid velocity is strictly subsonic. While this is not a hard requirement, we will see that for certain physical problems, and especially when parts of the problem need to be treated implicitly, this is a very desirable feature of the ideal FVS that we find below.

In the subsequent three Sub-sections we explore three possible flux vector splittings for the MHD system.

\section{II.1) Analysis of FVS for the MHD system that Permits Advection of Magnetic Energy}

We can formally write the hyperbolic system as $\partial_{t} \mathbf{U}+\partial_{x} \mathbf{F}=0$ so that the flux can be split as $\mathbf{F}=\mathbf{F}_{a}+\mathbf{F}_{p}$ where $\mathbf{F}_{a}$ is the advective flux and $\mathbf{F}_{p}$ is the pressure flux. Even if the pressure flux may have contributions from the magnetic field, it is still a nomenclatural simplification that we follow throughout this paper. Consequently, for this paper we will refer to the part of the flux vector that is not advected with the fluid velocity as the pressure sub-system. The first flux vector splitting that we try is very, very close to the original TV-FVS. It is given by 


$$
\mathbf{F}_{a}=\mathrm{v}_{x}\left(\begin{array}{c}
\rho \\
\rho \mathrm{v}_{x} \\
\rho \mathrm{v}_{y} \\
\rho \mathrm{v}_{z} \\
\rho \mathbf{v}^{2} / 2+\mathbf{B}^{2} / 4 \pi \\
0 \\
\mathrm{~B}_{y} \\
\mathrm{~B}_{z}
\end{array}\right) \quad ; \quad \mathbf{F}_{p}=\left(\begin{array}{c}
0 \\
\mathrm{P}+\mathbf{B}^{2} / 8 \pi-\mathrm{B}_{x}^{2} / 4 \pi \\
-\mathrm{B}_{x} \mathrm{~B}_{y} / 4 \pi \\
-\mathrm{B}_{x} \mathrm{~B}_{z} / 4 \pi \\
\gamma \mathrm{P}_{x} /(\gamma-1)-\mathrm{B}_{x}(\mathbf{v} \cdot \mathbf{B}) / 4 \pi \\
0 \\
-\mathrm{v}_{y} \mathrm{~B}_{x} \\
-\mathrm{v}_{z} \mathrm{~B}_{x}
\end{array}\right)
$$

In some sense, this is closest to the original TV-FVS because the full kinetic energy as well as all the quadratic parts of the magnetic energy are advected. Notice that for the pressure sub-system the density and $\mathrm{x}$-component of the magnetic field will be non-evolutionary. Consequently, the pressure sub-system always yields a $6 \times 6$ sub-system.

Written in conservation form the pressure sub-system from the FVS in eqn. (2.2) becomes

$$
\frac{\partial}{\partial t}\left(\begin{array}{c}
\rho \\
\rho \mathrm{v}_{x} \\
\rho \mathrm{v}_{y} \\
\rho \mathrm{v}_{z} \\
\varepsilon \\
\mathrm{B}_{x} \\
\mathrm{~B}_{y} \\
\mathrm{~B}_{z}
\end{array}\right)+\frac{\partial}{\partial x}\left(\begin{array}{c}
0 \\
\mathrm{P}+\mathrm{B}^{2} / 8 \pi-\mathrm{B}_{x}^{2} / 4 \pi \\
-\mathrm{B}_{x} \mathrm{~B}_{y} / 4 \pi \\
-\mathrm{B}_{x} \mathrm{~B}_{z} / 4 \pi \\
0 \\
0 \mathrm{v}_{x} /(\gamma-1)-\mathrm{B}_{x}(\mathbf{v} \cdot \mathbf{B}) / 4 \pi \\
-\mathrm{v}_{y} \mathrm{~B}_{x} \\
-\mathrm{v}_{z} \mathrm{~B}_{x}
\end{array}\right)=0
$$

It yields the following $6 \times 6$ sub-system 


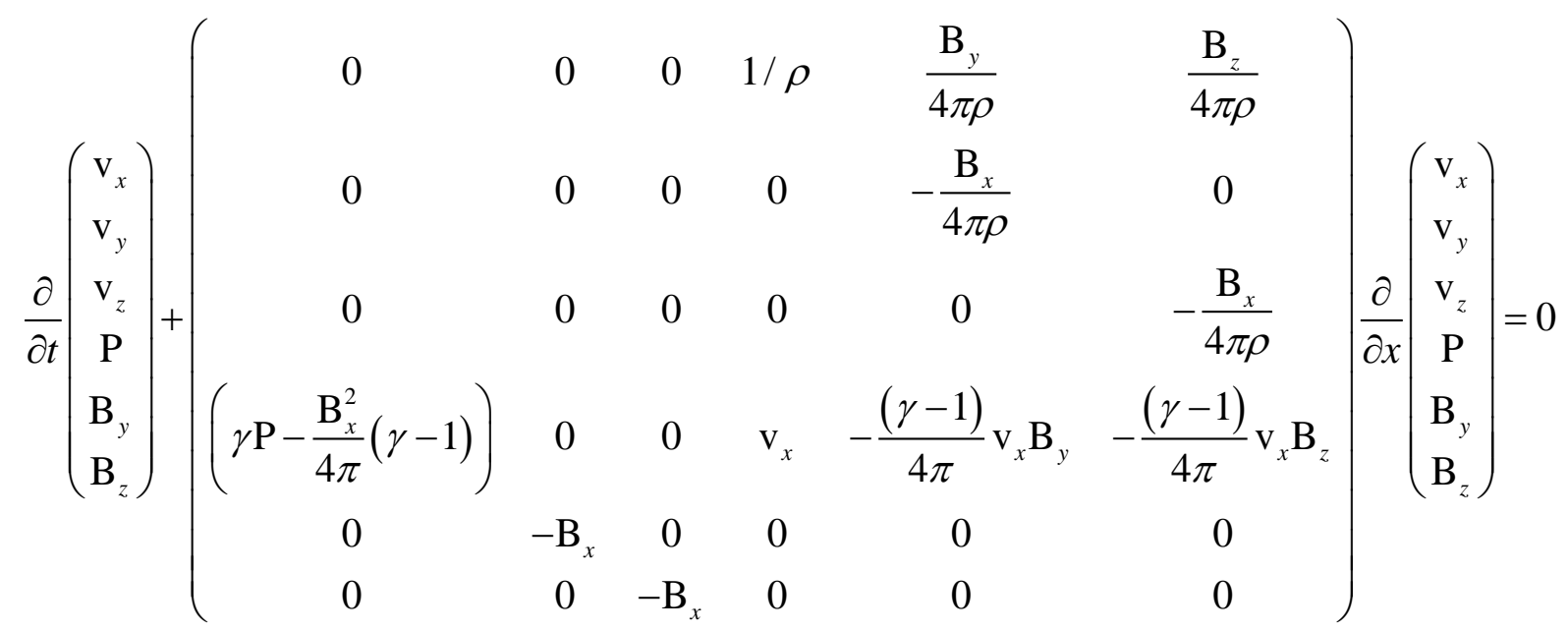

Analyzing the characteristic matrix in eqn. (2.4) tells us that $-\left|\mathrm{B}_{x}\right| / \sqrt{4 \pi \rho}$ will be a double root of the system and so will $\left|\mathrm{B}_{x}\right| / \sqrt{4 \pi \rho}$. We can recognize from this that the Alfven speeds in the $\mathrm{x}$-direction, given by $\pm\left|\mathrm{B}_{x}\right| / \sqrt{4 \pi \rho}$, are degenerate roots of the pressure system. But the fact that we have double roots for each of these Alfven waves implies that the analogue of the slow magnetosonic waves have become degenerate with the Alfven waves. Even so, it is easy to extract eigenvectors for the Alfven waves because those waves have polarization that is transverse to the original orientation of the $y$ - and $z$-directional magnetic fields. Alfven wave eigenvectors that propagate with Alfven speed $\mathrm{V}_{A x} \equiv\left|\mathrm{B}_{x}\right| / \sqrt{4 \pi \rho}$ will be denoted with a "+" subscript; Alfven wave eigenvectors that propagate with speed $-\mathrm{V}_{A x}$ will be denoted with a "_-" subscript. For the right Alfven wave eigenvectors we have

$$
\mathrm{R}_{A \pm}=\left[\begin{array}{llll}
0, & \pm \operatorname{sgn}\left(\mathrm{B}_{x}\right) \beta_{z}, \quad \mp \operatorname{sgn}\left(\mathrm{B}_{x}\right) \beta_{y}, \quad 0, \quad-\sqrt{4 \pi \rho} \beta_{z}, \quad \sqrt{4 \pi \rho} \beta_{y}
\end{array}\right]^{T}
$$

and for the orthonormalized left Alfven wave eigenvectors we have

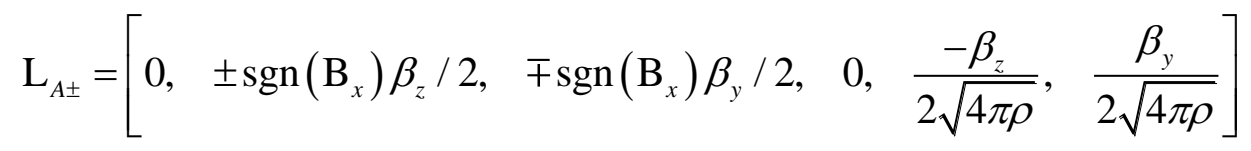

In the above equations, we define 
$\beta_{y} \equiv \mathrm{B}_{y} / \sqrt{\mathrm{B}_{y}^{2}+\mathrm{B}_{z}^{2}} \quad ; \quad \beta_{z} \equiv \mathrm{B}_{z} / \sqrt{\mathrm{B}_{y}^{2}+\mathrm{B}_{z}^{2}}$

While it may be interesting to study the analogues of the slow magnetosonic waves, subsequent analysis will show such a study to be useless for the FVS that we have chosen in eqn. (2.2). For that reason, we do not explore this direction further.

The remaining two eigenvalues of the characteristic matrix in eqn. (2.4) can be deemed to be closest in spirit to fast magnetosonic waves. They satisfy the quadratic equation

$\lambda^{2}-\mathrm{v}_{x} \lambda-\left(c_{s}^{2}-\mathrm{V}_{A x}^{2}(\gamma-1)\right)=0$

where $c_{s} \equiv \sqrt{\gamma \mathrm{P} / \rho}$. The roots of this equation are given by

$\lambda=\frac{\mathrm{v}_{x} \pm \sqrt{\mathrm{v}_{x}^{2}+4\left(c_{s}^{2}-\mathrm{V}_{A x}^{2}(\gamma-1)\right)}}{2}$

When the gas pressure is much larger than the magnetic pressure, i.e. $\mathrm{P} \square \mathbf{B}^{2} / 8 \pi$, it is easy to see that eqn. (2.9) guarantees that the fluid velocity is subsonic relative to the analogues of the fast magnetosonic waves. Such a gas pressure dominated situation prevails in the convective interiors of stars like the Sun. So we see that there are indeed systems of equations where the proposed FVS from this Sub-section might have some utility. (However, we will soon find a better FVS.) Notice, however, that in magnetic fusion systems like tokamaks or spheromaks, we might indeed have the opposite limit where $\mathrm{P} \sqcap \mathbf{B}^{2} / 8 \pi$. In such situations, it is even theoretically possible that eqn. (2.9) might indicate complex roots. In other words, for such systems the hyperbolic nature of the $6 \times 6$ sub-system in eqn. (2.4) is lost. For physical problems like tokamaks, the FVS discussed in this Sub-section becomes useless.

The exercise in this Sub-section has, however, been very instructive. Realize from eqn. (2.2) that we chose to advect the magnetic energy. This prevented the magnetic energy from playing a dominant role in dynamics of the energy equation. Since the magnetic energy enables the fast magnetosonic waves to move with the highest possible speeds, the consequence of not including the magnetic energy in the pressure sub-system was that the speed of the fast magnetosonic waves was reduced. We prefer to explore alternative flux vector splittings where 
some or all of the the magnetic energy in the energy equation (i.e. the fifth row in eqn. (2.1)) is included in the pressure sub-system.

\section{II.2) Analysis of FVS for the MHD system that is closest to Zha-Bilgen FVS}

The flux vector splitting for MHD that is closest to the ZB-FVS is given by

$$
\mathbf{F}_{Z B ; a}=\mathrm{v}_{x}\left(\begin{array}{c}
\rho \\
\rho \mathrm{v}_{x} \\
\rho \mathrm{v}_{y} \\
\rho \mathrm{v}_{z} \\
\varepsilon \\
0 \\
\mathrm{~B}_{y} \\
\mathrm{~B}_{z}
\end{array}\right) \quad ; \quad \mathbf{F}_{z B ; p}=\left(\begin{array}{c}
0 \\
\mathrm{P}+\mathrm{B}^{2} / 8 \pi-\mathrm{B}_{x}^{2} / 4 \pi \\
-\mathrm{B}_{x} \mathrm{~B}_{y} / 4 \pi \\
-\mathrm{B}_{x} \mathrm{~B}_{z} / 4 \pi \\
\left(\mathrm{P}+\mathbf{B}^{2} / 8 \pi\right) \mathrm{v}_{x}-\mathrm{B}_{x}(\mathbf{v} \cdot \mathbf{B}) / 4 \pi \\
0 \\
-\mathrm{v}_{y} \mathrm{~B}_{x} \\
-\mathrm{v}_{z} \mathrm{~B}_{x}
\end{array}\right)
$$

The fact that the system advects the vector of conserved variables makes it very convenient. Notice that we have included some portion of the magnetic energy in the fifth line of the pressure flux. But notice too that the total energy density in the advective flux still has some magnetic energy contribution that has not been included in the pressure sub-system. Thus the splitting in eqn. (2.10) represents a compromise between simplicity and physical intuition. Let us analyze how far such a compromise will take us.

As before, the evolutionary parts of the pressure sub-system can be written in primitive variables as a $6 \times 6$ system as follows 


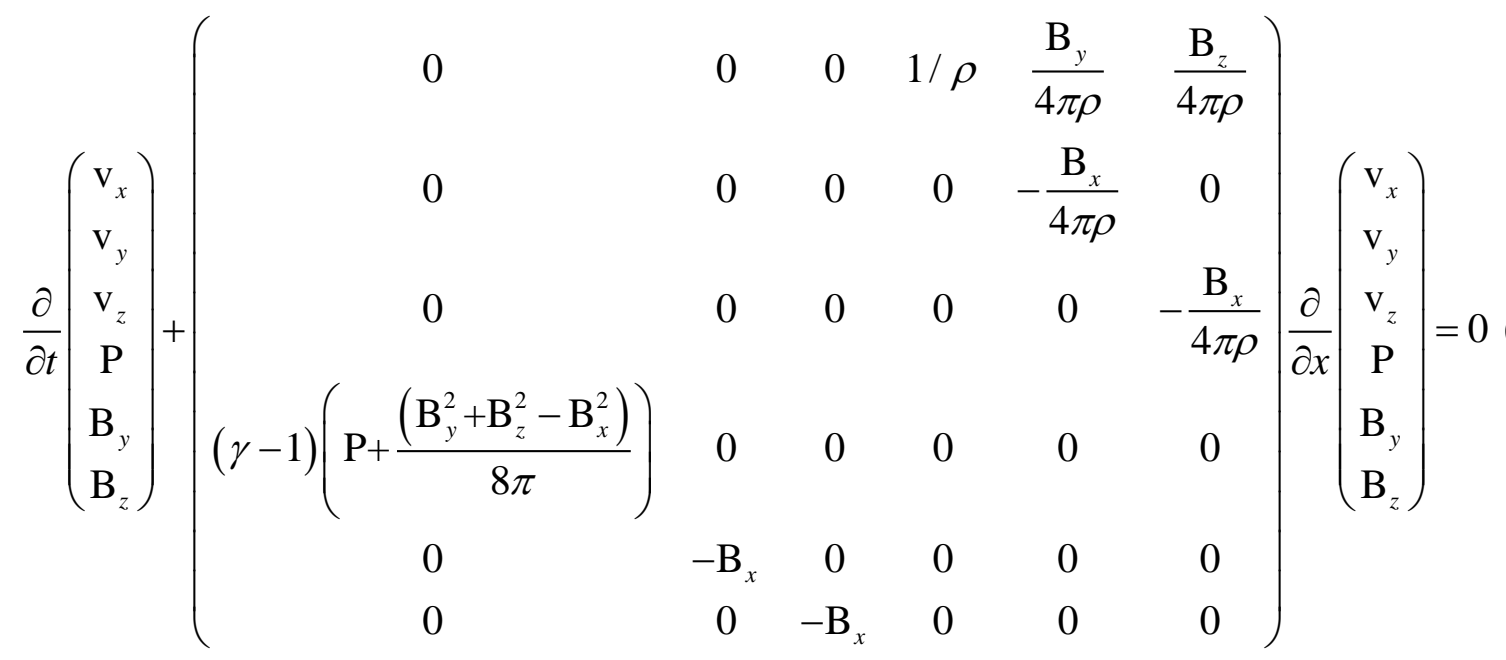

As before, analyzing the characteristic matrix in eqn. (2.11) tells us that $-\left|\mathrm{B}_{x}\right| / \sqrt{4 \pi \rho}$ will be a double root of the system and so will $\left|\mathrm{B}_{x}\right| / \sqrt{4 \pi \rho}$. In fact, the eigenvectors for the transverselypolarized Alfven waves are unchanged from their original expressions in eqns. (2.5), (2.6) and (2.7). This conforms to expectations because we only changed the fourth row in eqn. (2.11) compared to eqn. (2.4). That row pertains to the thermal energy (or pressure) equation. Since the pressure is left unchanged by Alfven waves, the structure of the Alfven waves is also unchanged.

The analogues of the fast magnetosonic waves now propagate with the speeds

$$
\lambda= \pm \sqrt{(\gamma-1)\left(c_{s}^{2} / \gamma+\left(\mathrm{V}_{A y}^{2}+\mathrm{V}_{A z}^{2}-\mathrm{V}_{A x}^{2}\right) / 2\right)}
$$

Here we define $\mathrm{V}_{A x} \equiv\left|\mathrm{B}_{x}\right| / \sqrt{4 \pi \rho} ; \mathrm{V}_{A y} \equiv\left|\mathrm{B}_{y}\right| / \sqrt{4 \pi \rho} ; \mathrm{V}_{A z} \equiv\left|\mathrm{B}_{z}\right| / \sqrt{4 \pi \rho}$. Notice that when the physical problem is dominated by gas pressure, the analogues of the fast magnetosonic waves from the ZB-FVS will always be the extremal waves in the Riemann fan that is formed by the pressure sub-system. In that gas-pressure dominated limit, the fluid velocity will always be subsonic relative to the analogues of the fast magnetosonic waves making it optimal for studies of stellar convection. However, notice too that in the opposite limit, when $\mathrm{P}<\mathbf{B}^{2} / 8 \pi$, it is easy to see that in at least some zones of a simulation we might have $\mathrm{B}_{y} \rightarrow 0$ and $\mathrm{B}_{z} \rightarrow 0$ with $\mathrm{P}<\mathrm{B}_{x}^{2} / 8 \pi$. In such situations, the roots in eqn. (2.12) can indeed become imaginary, obviating the hyperbolic nature of the system. Thus the ZB-FVS should be used very cautiously for MHD, 
and it should only be used when it is fully understood that the magnetized system is guaranteed (by the physics of the problem) to be gas-pressure dominated.

\section{II.3) Analysis of FVS for the MHD system that is closest to Toro-Vázquez FVS}

The flux vector splitting for MHD that is closest to the TV-FVS is given by

$$
\mathbf{F}_{T V ; a}=\mathrm{v}_{x}\left(\begin{array}{c}
\rho \\
\rho \mathrm{v}_{x} \\
\rho \mathrm{v}_{y} \\
\rho \mathrm{v}_{z} \\
\rho \mathbf{v}^{2} / 2 \\
0 \\
\mathrm{~B}_{y} \\
\mathrm{~B}_{z}
\end{array}\right) \quad ; \quad \mathbf{F}_{T V ; p}=\left(\begin{array}{c}
0 \\
\mathrm{P}+\mathbf{B}^{2} / 8 \pi-\mathrm{B}_{x}^{2} / 4 \pi \\
-\mathrm{B}_{x} \mathrm{~B}_{y} / 4 \pi \\
-\mathrm{B}_{x} \mathrm{~B}_{z} / 4 \pi \\
\left(\gamma \mathrm{P} /(\gamma-1)+\mathbf{B}^{2} / 4 \pi\right) \mathrm{v}_{x}-\mathrm{B}_{x}(\mathbf{v} \cdot \mathbf{B}) / 4 \pi \\
0 \\
-\mathrm{v}_{y} \mathrm{~B}_{x} \\
-\mathrm{v}_{z} \mathrm{~B}_{x}
\end{array}\right)
$$

Notice from the fifth component of the pressure flux in eqn. (2.13) that we have brought the full extent of the gas pressure as well as the magnetic pressure over into the pressure sub-system. This is desirable because it will also help in increasing the speeds of the analogues of the fast magnetosonic waves.

As before, the evolutionary parts of the pressure sub-system can be written in primitive variables as a $6 \times 6$ system as follows

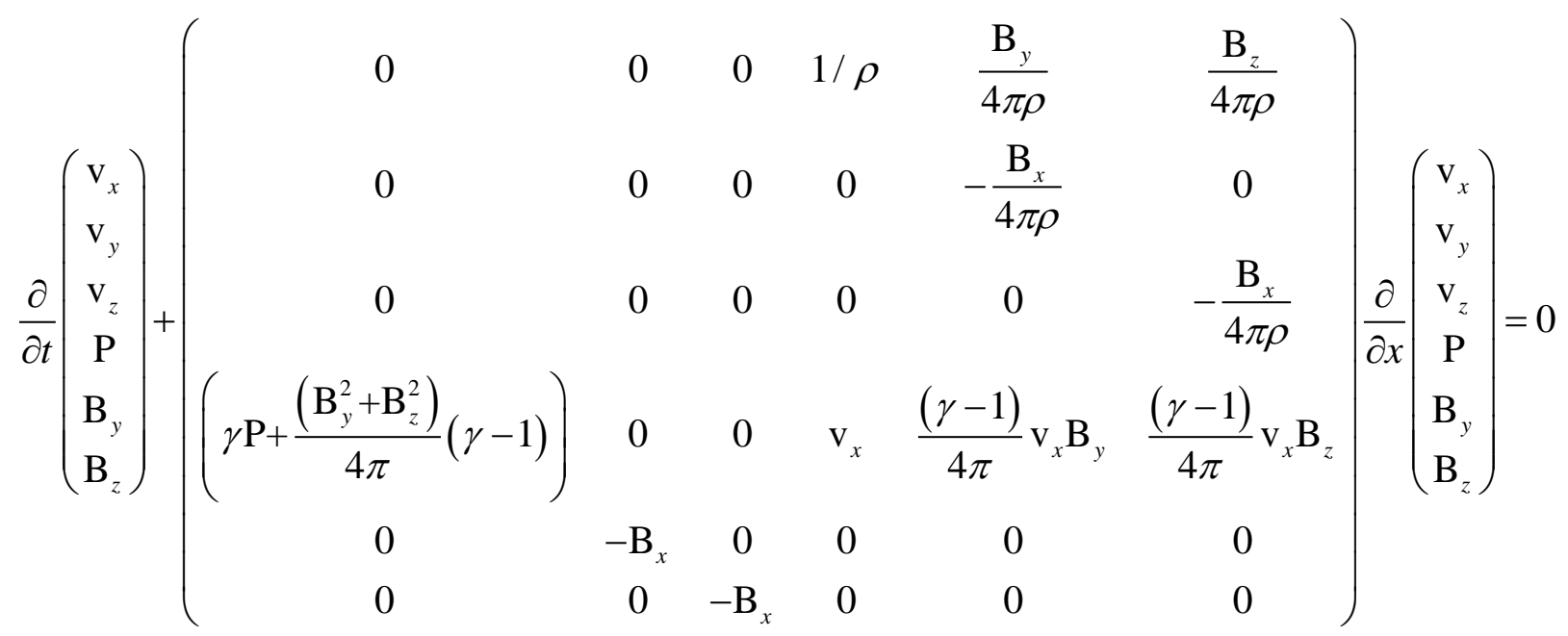


As before, analyzing the characteristic matrix in eqn. (2.14) tells us that $-\left|\mathrm{B}_{x}\right| / \sqrt{4 \pi \rho}$ will be a double root of the system and so will $\left|\mathrm{B}_{x}\right| / \sqrt{4 \pi \rho}$. In fact, the eigenvectors for the transverselypolarized Alfven waves are unchanged from their original expressions in eqns. (2.5), (2.6) and (2.7). This conforms to expectations.

The remaining two eigenvalues of the characteristic matrix in eqn. (2.14) can be deemed to be closest in spirit to fast magnetosonic waves. They satisfy the quadratic equation

$\lambda^{2}-\mathrm{v}_{x} \lambda-\left(c_{s}^{2}+(\gamma-1)\left(\mathrm{V}_{A y}^{2}+\mathrm{V}_{A z}^{2}\right)\right)=0$

The roots of this equation are given by

$\lambda=\frac{\mathrm{v}_{x} \pm \sqrt{\mathrm{v}_{x}^{2}+4\left(c_{s}^{2}+(\gamma-1)\left(\mathrm{V}_{A y}^{2}+\mathrm{V}_{A z}^{2}\right)\right)}}{2}$

Two very pleasant features of the above equation are worth pointing out. First, the fast magnetosonic waves always move supersonically relative to the fluid flow. This guarantees that the fluid velocity will always be subsonic relative to the Riemann fan of the pressure sub-system. This is true even in situations where magnetic pressure dominates. Second, even when the magnetic pressure dominates over the gas pressure (i.e., in the perverse situation that seemed to defeat the two previous attempts at obtaining a FVS for MHD) the above equation guarantees that the fast magnetosonic waves always form a hyperbolic system.

Notice though that it is not always guaranteed that the speeds stemming from eqn. (2.16) are always greater than the fast magnetosonic speed of the actual MHD system in eqn. (2.1). (This is also true for the TV-FVS for Euler flow where it cannot always be guaranteed that $\left(\mathrm{v}_{x}+\sqrt{\mathrm{v}_{x}^{2}+4 c_{s}^{2}}\right) / 2 \geq \mathrm{v}_{x}+c_{s}$ especially when $\mathrm{v}_{x}>0$.) Thus the speeds in eqn. (2.16) do not bracket the actual fast magnetosonic speeds for the MHD system. Also notice that for MHD, the speeds in eqn. (2.16) may not be the extremal speeds of the Riemann fan for the pressure subsystem. To see that, imagine a magnetic pressure dominated situation where $\mathrm{B}_{y} \rightarrow 0$ and $\mathrm{B}_{z} \rightarrow 0$ with $\mathrm{P}<\mathrm{B}_{x}^{2} / 8 \pi$. In such situations, it is possible that the Alfven speed, $\mathrm{V}_{A x}$, in the $\mathrm{x}-$ 
direction could be larger than the extremal wave speeds for the analogue of the fast magnetosonic waves from eqn. (2.16). In other words, the ordered wave foliation that prevails for the full MHD system can indeed be violated by the pressure sub-system. (Please recall that for the full MHD system in eqn. (2.1) the Alfven wave speeds are always bounded by the fast magnetosonic wave speeds; and the slow magnetosonic wave speeds are always bracketed by the Alfven wave speeds.) This deficiency in the foliation of waves for the pressure sub-system can have an impact on the design of a suitable Riemann solver for the TV-FVS for the MHD system.

\section{III) Riemann Solvers for MHD based on the Flux Vector Splitting}

Having identified an optimal FVS for the MHD system, we develop a Riemann solver that is based on it. Our focus for most of this section will be on the pressure sub-system because it provides the velocity with which the advection sub-system is to be chosen. Any Riemann solver has to rely on an identification of the extremal speeds in the Riemann fan. This requires a knowledge of the eigenvalues (but not necessarily all the eigenvectors) of the linearized Riemann solver. If we want to design a Riemann solver for the pressure sub-system for MHD, we will therefore have to linearize the MHD pressure sub-system and minimally obtain its eigenvalues. For that reason, Sub-section III.1 presents a linearization of the pressure sub-system for the FVS of the MHD equations.

The next step consists of designing a Riemann solver for the pressure sub-system associated with the MHD equations. To that end, we follow Dumbser and Balsara [24] and Balsara et al. [15] in realizing that not all the waves in the MHD system need to be followed exactly, Indeed, we are mostly interested in the entropy waves and Alfven waves because they carry the most information in an MHD simulation. For that reason, in Sub-section III.2 we present an HLLEM Riemann solver for the pressure sub-system which retains entropy wave and Alfven wave information. The design of this HLLEM Riemann solver is patterned after Dumbser and Balsara [24] and Balsara et al. [15]. Once the HLLEM Riemann solver is developed for the pressure sub-system, Sub-section III.3 presents the entire FVS-based Riemann solver for MHD.

\section{III.1) Wavespeeds from the Linearized Riemann Solver for the Pressure Sub-system}

Our mainstay solution technique will be based on the HLLEM Riemann solver, which is predicated on the HLL Riemann solver. Optimally, the HLL Riemann solver needs to obtain its 
extremal speeds from a Roe-type Riemann solver and the HLLEM Riemann solver requires the eigenvectors associated with the intermediate speeds. For this reason, we present a full linearization of the MHD Riemann problem for finite jumps between the left and right flow variables. Linearized Riemann solvers have been designed for the full MHD system by Brio and Wu [18], Cargo and Gallice [19] and Balsara [2]. The latter two papers rely on the properly normalized eigenvectors found by Roe and Balsara [48]. We will liberally make use of the linearizations that are known to work for the full MHD equations. The MHD flux is not homogeneous in terms of the conserved variables, as a result the Roe-averages for the magnetic field represent a little bit of an inspired guess that is designed to preserve the structure of the MHD equations. Consequently, the best linearizations for MHD are not based on systematic derivations like Roe and Pike [47] or Glaister [29]. The pressure sub-system from Sub-section II.3 for MHD can be written as

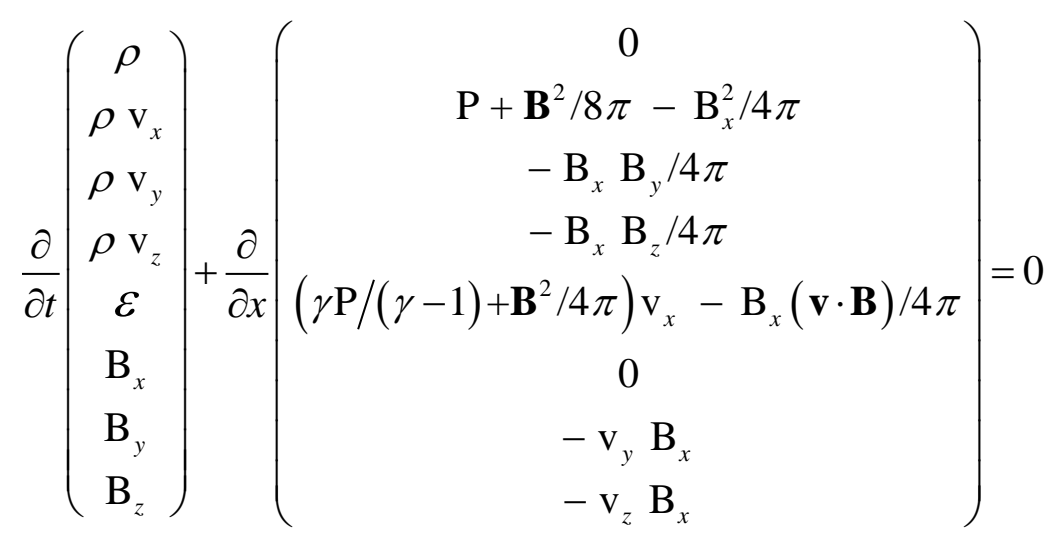

Notice that the density and the X-component of the magnetic field are not evolutionary in eqn. (3.1). In our notation the left and right state variables will carry subscripts of " $L$ " and " $R$ " respectively. We define our Roe-averaged density and velocities in the usual way by

$$
\begin{aligned}
& \rho^{*} \equiv \sqrt{\rho_{L} \rho_{R}} ; \overline{\mathrm{v}}_{\mathrm{x}} \equiv \frac{\sqrt{\rho_{L}} \mathrm{v}_{\mathrm{xL}}+\sqrt{\rho_{R}} \mathrm{v}_{\mathrm{x} R}}{\sqrt{\rho_{L}}+\sqrt{\rho_{R}}} ; \\
& \overline{\mathrm{v}}_{y} \equiv \frac{\sqrt{\rho_{L}} \mathrm{v}_{y L}+\sqrt{\rho_{R}} \mathrm{v}_{y R}}{\sqrt{\rho_{L}}+\sqrt{\rho_{R}}} ; \overline{\mathrm{v}}_{z} \equiv \frac{\sqrt{\rho_{L}} \mathrm{v}_{z L}+\sqrt{\rho_{R}} \mathrm{v}_{z R}}{\sqrt{\rho_{L}}+\sqrt{\rho_{R}}}
\end{aligned}
$$

The Roe-averaged magnetic fields are defined slightly differently by 


$$
\underline{\mathrm{B}}_{\mathrm{y}} \equiv \frac{\sqrt{\rho_{R}} \mathrm{~B}_{\mathrm{y} L}+\sqrt{\rho_{L}} \mathrm{~B}_{\mathrm{y} R}}{\sqrt{\rho_{L}}+\sqrt{\rho_{R}}} ; \quad \underline{\mathrm{B}}_{\mathrm{z}} \equiv \frac{\sqrt{\rho_{R}} \mathrm{~B}_{\mathrm{zL}}+\sqrt{\rho_{L}} \mathrm{~B}_{\mathrm{zR}}}{\sqrt{\rho_{L}}+\sqrt{\rho_{R}}}
$$

The fifth row of eqn. (3.1) enables us to identify the pressure and a free-stream enthalpy $H_{T V ; p}$ for the pressure sub-system as

$$
\mathrm{P}=(\gamma-1)\left[\varepsilon-\rho \frac{\mathbf{v}^{2}}{2}-\frac{\mathbf{B}^{2}}{8 \pi}\right] \quad ; \quad \rho H_{T V ; p}=\frac{\gamma \mathrm{P}}{(\gamma-1)}+\frac{\mathbf{B}^{2}}{4 \pi}=\gamma \varepsilon-\frac{\gamma}{2} \rho \mathbf{v}^{2}+(2-\gamma) \frac{\mathbf{B}^{2}}{8 \pi}
$$

Please notice that the enthalpy $H_{T V ; p}$ is somewhat different from the enthalpy that one would use for the full MHD equations. We define the Roe-averaged enthalpy as

$$
\bar{H}_{T V ; p} \equiv \frac{\sqrt{\rho_{L}} H_{T V ; p L}+\sqrt{\rho_{R}} H_{T V ; p R}}{\sqrt{\rho_{L}}+\sqrt{\rho_{R}}}
$$

These Roe-averaged quantities will be used to linearize the flux vector in eqn. (3.1) in the next paragraph.

Using the previously-defined Roe-averaged quantities, we can now write the change in all the flux terms in terms of the change in the conserved variables. The expressions that are hardest to derive are given below and can be verified by using a computer-based symbolic mathematics program.

$$
\begin{aligned}
& \Delta \mathrm{v}_{y}=-\frac{\overline{\mathrm{v}}_{\mathrm{y}}}{\rho^{*}} \Delta \rho+\frac{1}{\rho^{*}} \Delta\left(\rho \mathrm{v}_{y}\right) \\
& \Delta\left(\rho \mathrm{v}_{\mathrm{x}} \mathrm{v}_{\mathrm{y}}\right)=-\overline{\mathrm{v}}_{\mathrm{x}} \overline{\mathrm{v}}_{\mathrm{y}} \Delta \rho+\overline{\mathrm{v}}_{\mathrm{y}} \Delta\left(\rho \mathrm{v}_{\mathrm{x}}\right)+\overline{\mathrm{v}}_{\mathrm{x}} \Delta\left(\rho \mathrm{v}_{\mathrm{y}}\right) \\
& \Delta\left(\frac{\mathbf{B}^{2}}{2}\right)=\mathrm{X} \Delta \rho+\underline{\mathbf{B}} \cdot \Delta \mathbf{B} \quad ; \quad \mathrm{X} \equiv \frac{(\Delta \mathbf{B})^{2}}{2\left(\sqrt{\rho_{\mathrm{L}}}+\sqrt{\rho_{\mathrm{R}}}\right)^{2}} \\
& \Delta \mathrm{P}=(\gamma-1)\left[\left(\frac{\overline{\mathbf{v}}^{2}}{2}-\frac{\mathrm{X}}{4 \pi}\right) \Delta \rho-\overline{\mathbf{v}} \cdot \Delta(\rho \mathbf{v})+\Delta \varepsilon-\frac{1}{4 \pi} \underline{\mathbf{B}} \cdot \Delta \mathbf{B}\right]
\end{aligned}
$$




$$
\begin{aligned}
& \Delta \varepsilon=\left(\frac{\overline{\mathbf{v}}^{2}}{2}+\frac{\mathrm{X}}{4 \pi}\right) \Delta \rho+\rho^{*} \overline{\mathbf{v}} \cdot \Delta \mathbf{v}+\frac{\Delta \mathrm{P}}{(\gamma-1)}+\frac{1}{4 \pi} \underline{\mathbf{B}} \cdot \Delta \mathbf{B} \\
& \Delta\left(\rho H_{T V ; p}\right)=\left(\frac{\gamma}{2} \overline{\mathbf{v}}^{2}+(2-\gamma) \frac{\mathrm{X}}{4 \pi}\right) \Delta \rho-\gamma \overline{\mathbf{v}} \cdot \Delta(\rho \mathbf{v})+\gamma \Delta \varepsilon+\frac{(2-\gamma)}{4 \pi} \underline{\mathbf{B}} \cdot \Delta \mathbf{B} \\
& \Delta\left(\rho H_{T V ; p} \mathrm{v}_{x}\right)=-\overline{\mathrm{v}}_{x} \bar{H}_{T V ; p} \Delta \rho+\bar{H}_{T V ; p} \Delta\left(\rho \mathrm{v}_{x}\right)+\overline{\mathrm{v}}_{x} \Delta\left(\rho H_{T V ; p}\right) \\
& =\overline{\mathrm{v}}_{\mathrm{x}}\left(-\bar{H}_{T V ; p}+\frac{\gamma}{2} \overline{\mathbf{v}}^{2}+(2-\gamma) \frac{\mathrm{X}}{4 \pi}\right) \Delta \rho \\
& +\bar{H}_{T V ; p} \Delta\left(\rho \mathrm{v}_{\mathrm{x}}\right)-\gamma \overline{\mathrm{v}}_{\mathrm{x}} \overline{\mathbf{v}} \cdot \Delta(\rho \mathbf{v})+\gamma \overline{\mathrm{v}}_{\mathrm{x}} \Delta \boldsymbol{E}+\frac{(2-\gamma)}{4 \pi} \overline{\mathrm{v}}_{\mathrm{x}} \underline{\mathbf{B}} \cdot \Delta \mathbf{B} \\
& \Delta(\mathbf{v} \cdot \mathbf{B})=-\frac{1}{\rho^{*}}(\overline{\mathbf{v}} \cdot \underline{\mathbf{B}}) \Delta \rho+\frac{\mathrm{B}_{x}}{\rho^{*}} \Delta\left(\rho \mathbf{v}_{x}\right)+\frac{\underline{\mathrm{B}}_{y}}{\rho^{*}} \Delta\left(\rho \mathbf{v}_{y}\right)+\frac{\underline{\mathrm{B}}_{z}}{\rho^{*}} \Delta\left(\rho \mathbf{v}_{z}\right)+\overline{\mathbf{v}}_{y} \Delta \mathrm{B}_{y}+\overline{\mathrm{v}}_{z} \Delta \mathrm{B}_{z}
\end{aligned}
$$

From eqn. (3.8), please notice the introduction of the term " $\mathrm{X}$ ". It has no physical meaning and is just a reflection of the fact that our choice of Roe-averaged terms was slightly imperfect. However, at this point in time, better Roe-averages are not available for the MHD system in the literature. The system in eqn. (3.1) can be formally written in characteristic matrix form as $\partial_{t} \mathbf{U}+\mathrm{A}_{T V ; p} \partial_{x} \mathbf{U}=0$ where $\mathrm{A}_{T V ; p}$ is the characteristic matrix in conserved variables. When the left and right states differ by a finite jump, the Roe-averaged characteristic matrix is obtained by using eqns. (3.6) to (3.13) and is given by

$$
\overline{\mathrm{A}}_{T V ; p}=\left(\begin{array}{cccccccc}
0 & 0 & 0 & 0 & 0 & 0 & 0 & 0 \\
\frac{(\gamma-1)}{2} \overline{\mathbf{v}}^{2}+(2-\gamma) \frac{\mathrm{X}}{4 \pi} & -(\gamma-1) \overline{\mathrm{v}}_{x} & -(\gamma-1) \overline{\mathrm{v}}_{y} & -(\gamma-1) \overline{\mathrm{v}}_{z} & (\gamma-1) & 0 & (2-\gamma) \frac{\underline{\mathrm{B}}_{y}}{4 \pi} & (2-\gamma) \frac{\mathrm{B}_{z}}{4 \pi} \\
0 & 0 & 0 & 0 & 0 & 0 & -\frac{\mathrm{B}_{x}}{4 \pi} & 0 \\
0 & 0 & 0 & 0 & 0 & 0 & 0 & -\frac{\mathrm{B}_{x}}{4 \pi} \\
\delta_{51} & \delta_{52} & \delta_{53} & \delta_{54} & \delta_{55} & 0 & \delta_{57} & \delta_{58} \\
0 & 0 & 0 & 0 & 0 & 0 & 0 & 0 \\
\frac{\mathrm{B}_{x}}{\rho^{*}} \overline{\mathrm{v}}_{y} & 0 & -\frac{\mathrm{B}_{x}}{\rho^{*}} & 0 & 0 & 0 & 0 & 0 \\
\frac{\mathrm{B}_{x}}{\rho^{*}} \overline{\mathrm{v}}_{z} & 0 & 0 & -\frac{\mathrm{B}_{x}}{\rho^{*}} & 0 & 0 & 0 & 0
\end{array}\right)
$$


For the fifth row of the above matrix we have

$$
\begin{aligned}
& \delta_{51}=\overline{\mathrm{v}}_{\mathrm{x}}\left(-\bar{H}_{T V ; p}+\frac{\gamma}{2} \overline{\mathbf{v}}^{2}+(2-\gamma) \frac{\mathrm{X}}{4 \pi}\right)+\frac{\mathrm{B}_{x}}{4 \pi \rho^{*}}(\overline{\mathbf{v}} \underline{\mathbf{B}}) ; \delta_{52}=\bar{H}_{T V ; p}-\gamma \overline{\mathrm{v}}_{\mathrm{x}}^{2}-\frac{\mathrm{B}_{x}^{2}}{4 \pi \rho^{*}} ; \\
& \delta_{53}=-\gamma \overline{\mathrm{v}}_{x} \overline{\mathrm{v}}_{y}-\frac{\mathrm{B}_{x} \underline{\mathrm{B}}_{y}}{4 \pi \rho^{*}} ; \delta_{54}=-\gamma \overline{\mathrm{v}}_{x} \overline{\mathrm{v}}_{z}-\frac{\mathrm{B}_{x} \underline{\mathrm{B}}_{z}}{4 \pi \rho^{*}} ; \delta_{55}=\gamma \overline{\mathrm{v}}_{x} ; \\
& \delta_{57}=(2-\gamma) \overline{\mathrm{v}}_{x} \frac{\underline{\mathrm{B}}_{y}}{4 \pi}-\overline{\mathrm{v}}_{y} \frac{\mathrm{B}_{x}}{4 \pi} ; \delta_{58}=(2-\gamma) \overline{\mathrm{v}}_{x} \frac{\underline{\mathrm{B}}_{z}}{4 \pi}-\overline{\mathrm{v}}_{z} \frac{\mathrm{B}_{x}}{4 \pi}
\end{aligned}
$$

The eigenvalues and eigenvectors of the matrix in eqn. (3.14) will be the object of our study in the next few paragraphs.

We take our vector of primitive variables to be $\mathbf{V}=\left(\begin{array}{llllllllll}\rho & \mathrm{v}_{x} & \mathrm{v}_{y} & \mathrm{v}_{z} & \mathrm{P} & \mathrm{B}_{x} & \mathrm{~B}_{y} & \mathrm{~B}_{z}\end{array}\right)^{T}$ . It is also beneficial to relate the jump in the conserved variables, $\Delta \mathbf{U}$, to the jump in the primitive variables, $\Delta \mathbf{V}$. Using eqns. (3.6) and (3.10) we write

$$
\mathbf{C}=\frac{\Delta \mathbf{U}}{\Delta \mathbf{V}}=\left(\begin{array}{cccccccc}
1 & 0 & 0 & 0 & 0 & 0 & 0 & 0 \\
\overline{\mathrm{v}}_{x} & \rho^{*} & 0 & 0 & 0 & 0 & 0 & 0 \\
\overline{\mathrm{v}}_{y} & 0 & \rho^{*} & 0 & 0 & 0 & 0 & 0 \\
\overline{\mathrm{v}}_{z} & 0 & 0 & \rho^{*} & 0 & 0 & 0 & 0 \\
\frac{\overline{\mathbf{v}}^{2}}{2}+\frac{\mathrm{X}}{4 \pi} & \rho^{*} \overline{\mathrm{v}}_{x} & \rho^{*} \overline{\mathrm{v}}_{y} & \rho^{*} \overline{\mathrm{v}}_{z} & \frac{1}{(\gamma-1)} & 0 & \frac{\underline{\mathrm{B}}_{y}}{4 \pi} & \frac{\underline{\mathrm{B}}_{z}}{4 \pi} \\
0 & 0 & 0 & 0 & 0 & 1 & 0 & 0 \\
0 & 0 & 0 & 0 & 0 & 0 & 1 & 0 \\
0 & 0 & 0 & 0 & 0 & 0 & 0 & 1
\end{array}\right)
$$

The above matrix is almost lower-triangular and easily inverted; consequently, it is simple to evaluate $\mathrm{C}^{-1}$. It is explicitly written as. 


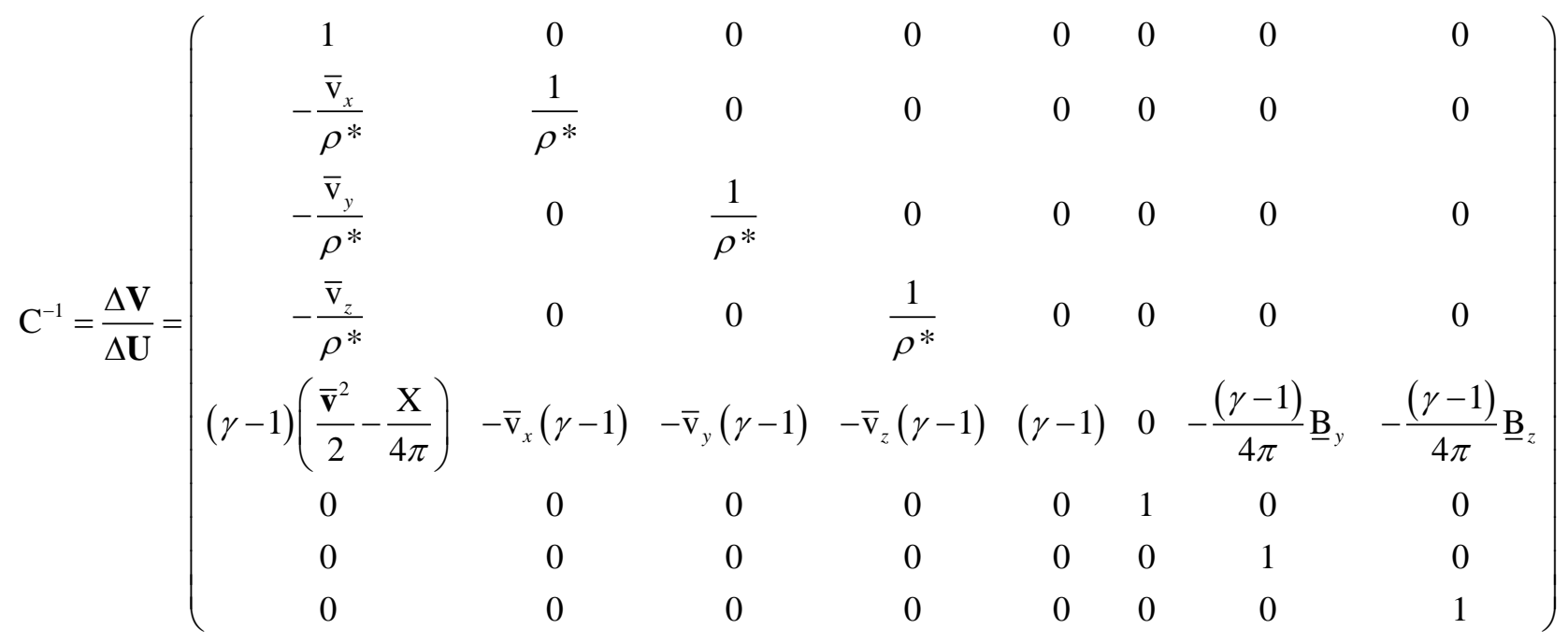

Eqns. (3.14), (3.16) and (3.17) enable us to obtain the Roe-averaged characteristic matrix in primitive variables as $\overline{\mathrm{A}}_{T V ; p}^{\text {prim }} \equiv \mathrm{C}^{-1} \overline{\mathrm{A}}_{T V ; p} \mathrm{C}$. This matrix is very useful for obtaining eigenvalues and eigenvectors. For $\overline{\mathrm{A}}_{T V ; p}^{\text {prim }}$ we get

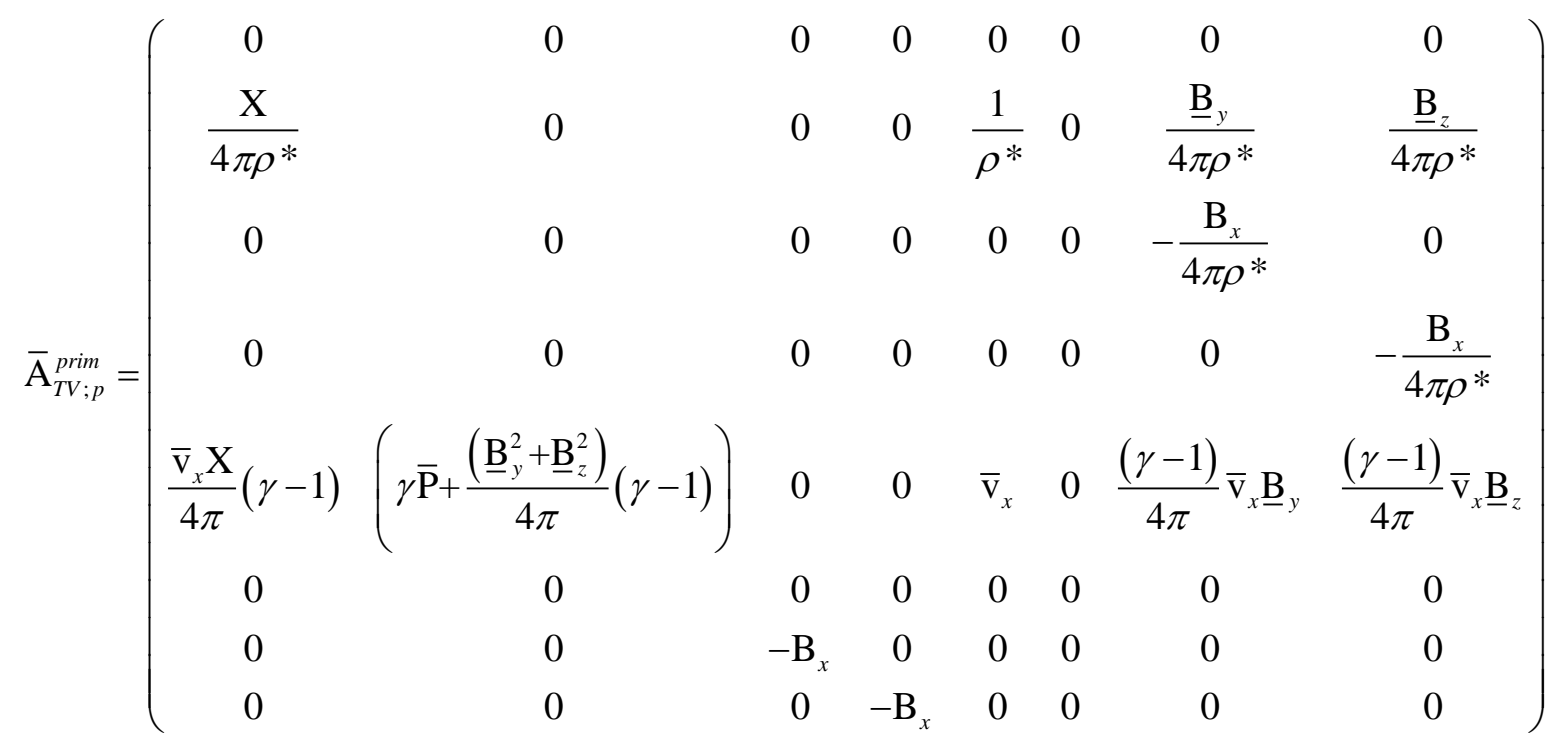

It is much easier to obtain the eigenvalues and eigenvectors of the characteristic matrix using eqn. (3.18). The eigenvectors for the conserved variables can be obtained by suitable multiplications of the matrix $\mathrm{C}$ or $\mathrm{C}^{-1}$ in eqns. (3.16) or (3.17). 
We now focus on deriving the eigenvalues and eigenvectors of the characteristic matrix in eqn. (3.18). To establish the concordance between eqn. (3.18) and eqn. (2.14) we need to define $\overline{\mathrm{P}}$ and the Alfven speeds in terms of Roe-averages. Doing this, we get

$$
\begin{aligned}
& \overline{\mathrm{P}}=\frac{(\gamma-1)}{\gamma} \rho^{*}\left(\overline{\mathrm{H}}_{T V ; p}-\frac{\mathrm{B}_{x}^{2}+\underline{\mathrm{B}}_{y}^{2}+\underline{\mathrm{B}}_{z}^{2}}{4 \pi \rho^{*}}\right) ; \bar{c}_{s}=\sqrt{\frac{\gamma \overline{\mathrm{P}}}{\rho^{*}}} ; \underline{\mathrm{B}}_{\perp}=\sqrt{\underline{\mathrm{B}}_{y}^{2}+\underline{\mathrm{B}}_{z}^{2}} ; \\
& \overline{\mathrm{V}}_{A x}=\frac{\left|\mathrm{B}_{x}\right|}{\sqrt{4 \pi \rho^{*}}} ; \overline{\mathrm{V}}_{A y}=\frac{\left|\underline{\mathrm{B}}_{y}\right|}{\sqrt{4 \pi \rho^{*}}} ; \overline{\mathrm{V}}_{A z}=\frac{\left|\underline{\mathrm{B}}_{z}\right|}{\sqrt{4 \pi \rho^{*}}} ; \bar{\beta}_{y}=\frac{\underline{\mathrm{B}}_{y}}{\underline{\mathrm{B}}_{\perp}} ; \bar{\beta}_{z}=\frac{\underline{\mathrm{B}}_{z}}{\underline{\mathrm{B}}_{\perp}}
\end{aligned}
$$

Please compare the definition of $\overline{\mathrm{P}}$ in eqn. (3.19) to eqn. (3.4) to see the similarities. The eigenvalues of eqn. (3.18) are also easily found. The Alfven and slow magnetosonic wave speeds again have a double degeneracy for the pressure sub-system, just like they did in Sub-section II.3. The fast magnetosonic wave speeds can also be written in a form that looks analogous to eqn. (2.16). We therefore write the eight eigenvalues as

$$
\begin{aligned}
& \lambda_{1}=\frac{\overline{\mathrm{V}}_{x}-\sqrt{\overline{\mathrm{V}}_{x}^{2}+4\left(\bar{c}_{s}^{2}+(\gamma-1)\left(\overline{\mathrm{V}}_{A y}^{2}+\overline{\mathrm{V}}_{A z}^{2}\right)\right)}}{2} ; \lambda_{2}=\lambda_{3}=-\overline{\mathrm{V}}_{A x} ; \lambda_{4}=\lambda_{5}=0 ; \\
& \lambda_{6}=\lambda_{7}=\overline{\mathrm{V}}_{A x} ; \lambda_{8}=\frac{\overline{\mathrm{V}}_{x}+\sqrt{\overline{\mathrm{V}}_{x}^{2}+4\left(\bar{c}_{s}^{2}+(\gamma-1)\left(\overline{\mathrm{V}}_{A y}^{2}+\overline{\mathrm{V}}_{A z}^{2}\right)\right)}}{2}
\end{aligned}
$$

Please compare the fast magnetosonic wave speeds in eqn. (3.20) with eqn. (2.16) to see the similarities. The similarities in the speeds of the Alfven and slow magnetosonic waves are obvious. Please also note that the ordering of the eigenvalues in eqn. (3.20) corresponds to the situation where the gas pressure exceeds the magnetic pressure. Consider situations where $\overline{\mathrm{V}}_{A y}$ and $\overline{\mathrm{V}}_{A z}$ are small but $\overline{\mathrm{V}}_{A x}$ is much larger than $\bar{c}_{s}$. In such situations we can certainly have $-\overline{\mathrm{V}}_{A x}$ as the most left-going speed or $\overline{\mathrm{V}}_{A x}$ as the most right-going speed. The eigenvalue ordering in eqn. (3.20) may not be preserved in such situations. It should, however, be observed that the pressure-subsystem is always subsonic, which enhances its utility for implicit formulations for MHD.

Let us now catalogue the complete right and left eigenvectors of eqn. (3.18). In the matrix expressions below, they are listed in the same order as the eigenvalues in eqn. (3.20). Alfven 
waves have a transverse polarization and we always place them between the fast and slow magnetosonic waves. The matrix of right eigenvectors for the pressure sub-system is given by

$$
\mathrm{R}_{T V ; p}^{p r i m}=\left(\begin{array}{cccccccc}
0 & 0 & 0 & 1 & 0 & 0 & 0 & 0 \\
1 & 0 & r_{23} & r_{24} & 0 & r_{26} & 0 & 1 \\
0 & -\operatorname{sgn}\left(\mathrm{B}_{x}\right) \bar{\beta}_{z} & \operatorname{sgn}\left(\mathrm{B}_{x}\right) \bar{\beta}_{y} & 0 & 0 & \operatorname{sgn}\left(\mathrm{B}_{x}\right) \bar{\beta}_{y} & \operatorname{sgn}\left(\mathrm{B}_{x}\right) \bar{\beta}_{z} & 0 \\
0 & \operatorname{sgn}\left(\mathrm{B}_{x}\right) \bar{\beta}_{y} & \operatorname{sgn}\left(\mathrm{B}_{x}\right) \bar{\beta}_{z} & 0 & 0 & \operatorname{sgn}\left(\mathrm{B}_{x}\right) \bar{\beta}_{z} & -\operatorname{sgn}\left(\mathrm{B}_{x}\right) \bar{\beta}_{y} & 0 \\
\rho^{*} \lambda_{1} & 0 & r_{53} & -\frac{\mathrm{X}}{4 \pi} & 0 & r_{56} & 0 & \rho^{*} \lambda_{8} \\
0 & 0 & 0 & 0 & 1 & 0 & 0 & 0 \\
0 & -\sqrt{4 \pi \rho^{*}} \bar{\beta}_{z} & \sqrt{4 \pi \rho^{*}} \bar{\beta}_{y} & 0 & 0 & -\sqrt{4 \pi \rho^{*}} \bar{\beta}_{y} & -\sqrt{4 \pi \rho^{*}} \bar{\beta}_{z} & 0 \\
0 & \sqrt{4 \pi \rho^{*}} \bar{\beta}_{y} & \sqrt{4 \pi \rho^{*}} \bar{\beta}_{z} & 0 & 0 & -\sqrt{4 \pi \rho^{*}} \bar{\beta}_{z} & \sqrt{4 \pi \rho^{*}} \bar{\beta}_{y} & 0
\end{array}\right)
$$

where we define

$$
\begin{aligned}
& r_{24}=\frac{(\gamma-2)}{\rho^{*} \lambda_{1} \lambda_{8}} \frac{\overline{\mathrm{v}}_{x} \mathrm{X}}{4 \pi} ; \\
& r_{23}=-\frac{\underline{\mathrm{B}}_{\perp}}{\sqrt{4 \pi \rho^{*}}}\left(\frac{(2-\gamma) \overline{\mathrm{V}}_{x}+\overline{\mathrm{V}}_{A x}}{\overline{\mathrm{V}}_{A x}\left(\overline{\mathrm{V}}_{x}+\overline{\mathrm{V}}_{A x}\right)+\lambda_{1} \lambda_{8}}\right) ; r_{53}=-\frac{\underline{\mathrm{B}}_{\perp} \rho^{*}}{\sqrt{4 \pi \rho^{*}}}\left(\frac{\lambda_{1} \lambda_{8}+(\gamma-1) \overline{\mathrm{V}}_{x} \overline{\mathrm{V}}_{A x}}{\overline{\mathrm{V}}_{A x}\left(\overline{\mathrm{V}}_{x}+\overline{\mathrm{V}}_{A x}\right)+\lambda_{1} \lambda_{8}}\right) ; \\
& r_{26}=\frac{\underline{\mathrm{B}}_{\perp}}{\sqrt{4 \pi \rho^{*}}}\left(\frac{(2-\gamma) \overline{\mathrm{V}}_{x}-\overline{\mathrm{V}}_{A x}}{\lambda_{1} \lambda_{8}-\overline{\mathrm{V}}_{A x}\left(\overline{\mathrm{V}}_{x}-\overline{\mathrm{V}}_{A x}\right)}\right) ; r_{56}=\frac{\underline{\mathrm{B}}_{\perp} \rho^{*}}{\sqrt{4 \pi \rho^{*}}}\left(\frac{\lambda_{1} \lambda_{8}-(\gamma-1) \overline{\mathrm{V}}_{x} \overline{\mathrm{V}}_{A x}}{\lambda_{1} \lambda_{8}-\overline{\mathrm{V}}_{A x}\left(\overline{\mathrm{V}}_{x}-\overline{\mathrm{V}}_{A x}\right)}\right)
\end{aligned}
$$

The matrix of left, orthonormalized eigenvectors for the pressure sub-system is given by 


$$
\mathrm{L}_{T V ; p}^{\text {prim }}=\left(\begin{array}{cccccccc}
l_{11} & -\frac{\lambda_{8}}{\left(\lambda_{1}-\lambda_{8}\right)} & -\kappa_{1} \mathrm{~B}_{x} \underline{\mathrm{B}}_{y} & -\kappa_{1} \mathrm{~B}_{x} \underline{\mathrm{B}}_{z} & \frac{1}{\rho^{*}\left(\lambda_{1}-\lambda_{8}\right)} & 0 & \kappa_{1} \lambda_{1} \underline{\mathrm{B}}_{y} & \kappa_{1} \lambda_{1} \underline{\mathrm{B}}_{z} \\
0 & 0 & -\frac{\operatorname{sgn}\left(\mathrm{B}_{x}\right) \bar{\beta}_{z}}{2} & \frac{\operatorname{sgn}\left(\mathrm{B}_{x}\right) \bar{\beta}_{y}}{2} & 0 & 0 & \frac{-\bar{\beta}_{z}}{2 \sqrt{4 \pi \rho^{*}}} & \frac{\bar{\beta}_{y}}{2 \sqrt{4 \pi \rho^{*}}} \\
0 & 0 & \frac{\operatorname{sgn}\left(\mathrm{B}_{x}\right) \bar{\beta}_{y}}{2} & \frac{\operatorname{sgn}\left(\mathrm{B}_{x}\right) \bar{\beta}_{z}}{2} & 0 & 0 & \frac{\bar{\beta}_{y}}{2 \sqrt{4 \pi \rho^{*}}} & \frac{\bar{\beta}_{z}}{2 \sqrt{4 \pi \rho^{*}}} \\
0 & 0 & 0 & 0 & 0 & 0 & 0 & 0 \\
0 & 0 & \frac{\operatorname{sgn}\left(\mathrm{B}_{x}\right) \bar{\beta}_{y}}{2} & \frac{\operatorname{sgn}\left(\mathrm{B}_{x}\right) \bar{\beta}_{z}}{2} & 0 & 0 & -\frac{\bar{\beta}_{y}}{2 \sqrt{4 \pi \rho^{*}}} & -\frac{\bar{\beta}_{z}}{2 \sqrt{4 \pi \rho^{*}}} \\
0 & \frac{\operatorname{sgn}\left(\mathrm{B}_{x}\right) \bar{\beta}_{z}}{2} & -\frac{\operatorname{sgn}\left(\mathrm{B}_{x}\right) \bar{\beta}_{y}}{2} & 0 & 0 & \frac{-\bar{\beta}_{z}}{2 \sqrt{4 \pi \rho^{*}}} & \frac{\bar{\beta}_{y}}{2 \sqrt{4 \pi \rho^{*}}} \\
l_{81}-\frac{\lambda_{1}}{\left(\lambda_{8}-\lambda_{1}\right)} & -\kappa_{8} \mathrm{~B}_{x} \underline{\mathrm{B}}_{y} & -\kappa_{8} \mathrm{~B}_{x} \underline{\mathrm{B}}_{z} & \frac{1}{\rho^{*}\left(\lambda_{8}-\lambda_{1}\right)} & 0 & \kappa_{8} \lambda_{8} \underline{\mathrm{B}}_{y} & \kappa_{8} \lambda_{8} \underline{\mathrm{B}}_{z}
\end{array}\right)
$$

where we define

$$
\begin{aligned}
& l_{11}=\frac{1}{\rho^{*} \lambda_{1}\left(\lambda_{1}-\lambda_{8}\right)} \frac{\mathrm{X}}{4 \pi}\left(\overline{\mathrm{v}}_{x}(\gamma-1)-\lambda_{8}\right) ; \kappa_{1}=\frac{1}{\left(\lambda_{1}-\lambda_{8}\right)}\left(\frac{\lambda_{8}-(\gamma-1) \overline{\mathrm{v}}_{x}}{\mathrm{~B}_{x}^{2}-4 \pi \rho^{*} \lambda_{1}^{2}}\right) \\
& l_{81}=\frac{1}{\rho^{*} \lambda_{8}\left(\lambda_{8}-\lambda_{1}\right)} \frac{\mathrm{X}}{4 \pi}\left(\overline{\mathrm{v}}_{x}(\gamma-1)-\lambda_{1}\right) ; \kappa_{8}=\frac{1}{\left(\lambda_{8}-\lambda_{1}\right)}\left(\frac{\lambda_{1}-(\gamma-1) \overline{\mathrm{v}}_{x}}{\mathrm{~B}_{x}^{2}-4 \pi \rho^{*} \lambda_{8}^{2}}\right)
\end{aligned}
$$

Some very interesting observations can be made about the eigenvectors. Notice from eqn. (3.21) that in the limit where the magnetic field goes to zero, the fast magnetosonic waves become the analogues of the sound waves identified in Toro and Vázquez. Recall too that the sound waves in TV-FVS only induce fluctuations in the pressure and longitudinal velocity for the pressure subsystem. Likewise, eqn. (3.21) shows us that the fast magnetosonic waves only correspond to fluctuations in the pressure and longitudinal velocity for the pressure sub-system. This is a very surprising feature of this FVS for MHD. A typical fast magnetosonic wave for the full MHD system will also induce fluctuations in the transverse velocities and magnetic fields. The FVS for MHD, therefore, has some remarkable properties which might eventually be exploited for producing implicit schemes for MHD. From eqn. (3.21) it is also interesting to observe from our pressure sub-system that the Alfven waves have a transverse polarization while the slow 
magnetosonic waves have a polarization that lies along the magnetic field structure. The full MHD equations also show this same trend. It is, therefore, heartening to see much of the familiar physics reflected in the new eigenvectors that we have found for the pressure sub-system for MHD.

\section{III.2) HLLEM Riemann Solver for the Pressure Sub-system}

Eqn. (3.1) defines the pressure sub-system. The easiest way to obtain a serviceable flux vector splitting-based Riemann solver for MHD is the following. First, treat eqn. (3.1) as a conservation law and use an HLL Riemann solver to obtain the resolved state and flux for the pressure sub-system. This is easy because eqn. (2.16), as well as the Alfven speeds, can be evaluated to obtain the wave speeds in the left and right states. Using eqns. (3.2), (3.3), (3.5) and (3.19) we can evaluate the Roe-averaged state for the given left and right states. Eqn. (3.20) then enables us to obtain the extremal speeds for the Roe-averaged states. Once these signal speeds are obtained, we can obtain the extremal speeds for the HLL Riemann solver. Consequently, we can obtain the resolved HLL state and HLL flux for the pressure sub-system. (Recall that the pressure sub-system always results in a subsonic Riemann fan. Also notice that the eigenvectors from eqn. (3.21) to (3.24) do not need to be evaluated if we are satisfied with an HLL Riemann solver.) Second, using the resolved state for the pressure sub-system, find the $\mathrm{x}$-velocity. This is the $\mathrm{x}$-velocity that pre-multiplies the advected flux from the advection sub-system in eqn. (2.13). If the x-velocity in the resolved state is positive, build the rest of the advected flux using the left state. If the $\mathrm{x}$-velocity in the resolved state is negative, build the rest of the advected flux using the right state. Adding the advected flux from the advection sub-system to the HLL flux from the pressure sub-system gives us a serviceable FVS-based Riemann solver for MHD. We have constructed such a Riemann solver and found it to be robust and to work well for a range of test problems. However, such a Riemann solver will not preserve stationary contact discontinuities exactly on the mesh. Thus we must look further.

To preserve stationary contact discontinuities exactly, we look to the recent work on the HLLEM Riemann solver by Dumbser and Balsara [24]. This Riemann solver improves on the basic HLL Riemann solver by including the contribution from intermediate waves in an antidiffusive fashion so as to enhance the quality of the numerical HLL flux. The HLLEM Riemann solver, therefore, requires the evaluation of eigenvectors for the intermediate waves. When all 
intermediate waves are included, it becomes a complete Riemann solver. By intermediate waves we mean the Alfven waves, the entropy wave and the slow magnetosonic waves in eqns. (3.21) to (3.24). The explicit expressions for those eigenvectors are given in this paper. All details about the HLLEM Riemann solver are presented in Dumbser and Balsara [24] and we refer the reader to that paper for a thorough description. However, the HLLEM Riemann solver is still quite new. Therefore, in the next two paragraphs we operationally describe how the eigenvalues and eigenvectors from the Sub-section III.1 can be used to construct an HLLEM Riemann solver for the pressure sub-system for MHD.

Let the extremal speeds of the Riemann fan associated with the pressure sub-system be given by $S_{L}$ and $S_{R}$ and let $\lambda_{i}$ denote the wave speed of one of the intermediate waves with $i=2, \ldots, 7$. The subscripts " $\mathrm{L}$ " and " $\mathrm{R}$ " will, in general, identify the left and right states. The HLLEM Riemann solver then relies on evaluating a multiplicative factor $\delta_{i}$ for each wave family given by

$$
\delta_{i}=1-\frac{\lambda_{i}^{-}}{S_{L}}-\frac{\lambda_{i}^{+}}{S_{R}}
$$

This is eqn. (29) from Dumbser and Balsara [24]. Here we define $\lambda_{i}^{-}=\min \left(\lambda_{i}, 0\right)$ and $\lambda_{i}^{+}=\max \left(\lambda_{i}, 0\right)$. The term $\delta_{i}$ is an anti-diffusive term and enables us to reduce the diffusion as much as possible in the HLLEM Riemann solver without losing stability. We can then define a $6 \times 6$ diagonal matrix given by $\boldsymbol{\delta}=\operatorname{diag}\left\{\delta_{i}\right\}$. Dropping the first and last columns in eqn. (3.21) gives us an $8 \times 6$ matrix of right eigenvectors for the intermediate waves. Denote it by $\tilde{\mathrm{R}}_{T V ; p}^{\text {prim }}$. Using eqn. (3.16), we obtain an $8 \times 6$ matrix of the same right eigenvectors in conserved variables given by $\tilde{\mathrm{R}}_{T V ; p}=\mathrm{C} \tilde{\mathrm{R}}_{T V ; p}^{\text {prim }}$. Dropping the first and last rows in eqn. (3.23) gives us a $6 \times 8$ matrix of left eigenvectors for the intermediate waves. Denote it by $\tilde{\mathrm{L}}_{T V ; p}^{\text {prim }}$. Using eqn. (3.17), we obtain a $6 \times 8$ matrix of the same left eigenvectors in conserved variables given by $\tilde{\mathrm{L}}_{T V ; p}=\tilde{\mathrm{L}}_{T V ; p}^{\text {prim }} \mathrm{C}^{-1}$. Notice that the eigenvectors for the extremal waves, i.e. the fast waves, don't need to be evaluated. Their contributions are already accounted for by the shock jumps that are imposed at either end of the Riemann fan associated with the HLL Riemann solver. This 
completes our description of the matrices that will help us in constructing the HLLEM state and flux. We do that in the next paragraph.

In the HLLEM Riemann solver, the resolved state has a suitable linear variation within the Riemann fan. This linear variation also provides a built-in entropy fix. The resolved state that coincides with the zone boundary is obtained by setting $\xi=0$ in eqn. (26) of Dumbser and Balsara [24] and is given by

$$
\overline{\mathbf{U}}_{T V ; p ; z o n e-b d y}=\overline{\mathbf{U}}_{T V ; p ; H L L}-\phi\left(\frac{S_{R}+S_{L}}{S_{R}-S_{L}}\right) \tilde{\mathrm{R}}_{T V ; p} \boldsymbol{\delta} \tilde{\mathrm{L}}_{T V ; p}\left(\mathbf{U}_{R}-\mathbf{U}_{L}\right)
$$

Here $\mathbf{U}_{R}$ and $\mathbf{U}_{L}$ denote the right and left states in the Riemann problem and $\overline{\mathbf{U}}_{T V ; p ; H L L}$ is the resolved HLL state from the pressure sub-system in eqn. (3.1). Eqn. (3.26) can be further clarified by realizing that $\tilde{\mathrm{L}}_{T V ; p}\left(\mathbf{U}_{R}-\mathbf{U}_{L}\right)$ gives us eigenweights associated with the jump in the states; consequently, the term $\boldsymbol{\delta} \tilde{\mathrm{L}}_{T V ; p}\left(\mathbf{U}_{R}-\mathbf{U}_{L}\right)$ gives us a set of weights that should be used for the right eigenvectors. The parameter $\phi$ is a flattener parameter and its usage is described in Balsara [8]. In regions of smooth flow, $\phi=1$. In the vicinity of strong shocks it becomes zero, thus reverting back to an HLL Riemann solver which is more stabilizing near shocks. The state $\overline{\mathbf{U}}_{T V ; p ; z o n e-b d y}$ is indeed the HLLEM state obtained from the pressure sub-system that coincides with the zone boundary. The ratio of its second component to its first component indeed gives us the $\mathrm{x}$-velocity that pre-multiplies the advected flux for the advection sub-system in eqn. (2.13). We would indeed like to obtain the HLLEM flux corresponding to the state in eqn. (3.26). This is obtained by using eqn. (30) of Dumbser and Balsara [24]. It is given by

$$
\overline{\mathbf{F}}_{T V ; p ; H L L E M}=\overline{\mathbf{F}}_{T V ; p ; H L L}-\phi\left(\frac{S_{R} S_{L}}{S_{R}-S_{L}}\right) \tilde{\mathrm{R}}_{T V ; p} \boldsymbol{\delta} \tilde{\mathrm{L}}_{T V ; p}\left(\mathbf{U}_{R}-\mathbf{U}_{L}\right)
$$

Here $\overline{\mathbf{F}}_{T V ; p ; H L L}$ is the resolved HLL flux from the pressure sub-system in eqn. (3.1). The HLLEM flux for the pressure sub-system is given by $\overline{\mathbf{F}}_{T V ; p ; H L L E M}$. The role of the diagonal matrix $\boldsymbol{\delta}$ in supplying the anti-diffusive part of the HLLEM flux is easy to see. It has been further amplified 
in Appendix B of Dumbser and Balsara [24]. This completes our description of the HLLEM Riemann solver as it applies to the pressure sub-system for MHD.

In this paper the HLLEM Riemann solver will be used for solving the pressure system and obtaining a numerical flux contribution from it. The HLLEM Riemann solver presented here is a complete Riemann solver for the pressure sub-system - it accounts for all the intermediate waves that arise in the pressure sub-system. In the rare occasions that the ordered foliation of waves in eqn. (3.20) is violated, we recommend that one should revert back to an HLL Riemann solver.

It is also possible to obtain a significant simplification of the HLLEM Riemann solver described here. To achieve this simplification, we realize that most of the times we are only interested in improving the quality of the entropy wave and the Alfven waves. We can, therefore, define the $\tilde{\mathrm{R}}_{T V ; p}^{\text {prim }}$ matrix to be an $8 \times 3$ matrix of right eigenvectors obtained by taking just the second, fourth and seventh columns in eqn. (3.21). Likewise, we can define the $\tilde{\mathbf{L}}_{T V ; p}^{\text {prim }}$ matrix to be a $3 \times 8$ matrix of left eigenvectors obtained by taking just the second, fourth and seventh rows in eqn. (3.23). The matrix $\delta$ also becomes a $3 \times 3$ diagonal matrix obtained by considering just the two eigenvalues of the two Alfven waves and the eigenvalue of the entropy wave. The resulting HLLEM Riemann solver will not be complete. Yet it will be adequately sophisticated and will be able to retain the essential waves that we are interested in. Eqns. (3.26) and (3.27) still give us the HLLEM state and the HLLEM flux of the pressure sub-system. We have also experimented with such a Riemann solver and found it to produce results that are just as good as a full HLLEM Riemann solver. It was, therefore, set as our default HLLEM Riemann solver for all the test problems shown in this paper. The simplification achieved here also underscores the simplicity and versatility of the HLLEM Riemann solver of Dumbser and Balsara [24].

\section{III.3) FVS-based Riemann Solver for MHD}

Once the pressure sub-system is solved using the method described in Sub-section III.2, the solution of the advection sub-system is easy. Using the first and second components of $\overline{\mathbf{U}}_{T V ; p ; z n n e-b d y}$ from eqn. (3.26) we obtain an X-velocity $\tilde{\mathbf{v}}_{x ; T V ; p}=\left(\overline{\mathbf{U}}_{T V ; p ; z o n e-b d y}\right)_{2} /\left(\overline{\mathbf{U}}_{T V ; p ; z \text { zone-bdy }}\right)_{1}$.

We define the numerical flux from the advection sub-system as $\overline{\mathbf{F}}_{T V ; a}$ which is given by 


$$
\overline{\mathbf{F}}_{T V ; a}=\left\{\begin{array}{llllllll}
\tilde{\mathrm{v}}_{x ; T V ; p}\left(\rho_{L},\right. & \rho_{L} \mathrm{v}_{x L}, & \rho_{L} \mathrm{v}_{y L}, & \rho_{L} \mathrm{v}_{z L}, & \rho_{L} \mathbf{v}_{L}^{2} / 2, & 0 & \mathrm{~B}_{y L}, & \left.\mathrm{~B}_{z L}\right)^{T} \text { when } \tilde{\mathrm{v}}_{x ; T V ; p} \geq 0 \\
\tilde{\mathrm{v}}_{x ; T V ; p}\left(\rho_{R},\right. & \rho_{R} \mathrm{v}_{x R}, & \rho_{R} \mathrm{v}_{y R}, & \rho_{R} \mathrm{v}_{z R}, & \rho_{R} \mathbf{v}_{R}^{2} / 2, & 0 & \mathrm{~B}_{y R}, & \left.\mathrm{~B}_{z R}\right)^{T} \text { when } \tilde{\mathrm{v}}_{x ; T V ; p}<0
\end{array}\right.
$$

The subscripts " $L "$ and "R" describe the left and right states. Once the numerical flux from the advection sub-system is built, the final numerical flux for the entire MHD system is given by

$\overline{\mathbf{F}}_{T V ; a}+\overline{\mathbf{F}}_{T V ; p ; H L L E M}$. This completes our description of the flux vector splitting for the MHD system.

\section{IV) Results}

We present the results from several stringent one-dimensional test problems that highlight the capability of our FVS-based Riemann solver for MHD. All our results were obtained by using second order spatial reconstruction in primitive variables applied to the characteristic form of the MHD equations. An MC limiter was used for all the spatial reconstruction. The temporal update used an SSP-RK2 time stepping strategy from Shu and Osher [52]. The characteristic reconstruction as well as the SSP-RK2 timestepping, as they apply to MHD, have been described in Balsara [4]. Consequently, we do not repeat them here. The HLLEM Riemann solver was applied to the pressure sub-system where we retained just the contribution from the Alfven waves and the entropy wave, as described in the last paragraph of Sub-section III.2. A CFL of 0.8 was used for all the simulations, where the CFL was set by using just the regular MHD wavespeeds. In other words, the extremal speeds $S_{L}$ and $S_{R}$ from the pressure sub-system were not used in any way to regulate the timestep.

It is also worth mentioning that the strategy presented here also works very well for Euler flow. The HLLEM Riemann solver for the pressure sub-system should then include the entropy wave and the shear waves. The linearization from Sub-section III.1 is easily adapted to Euler flow and the eigenvectors of the Alfven waves naturally reduce to the eigenvectors of the shear waves. The corresponding eigenvectors are extremely easy to evaluate. An extremely efficient FVS-based Riemann solver is then obtained for Euler flow. The resulting FVS-based Riemann solver can preserve isolated, stationary contact discontinuities and shear waves on the mesh. 
Table I catalogues the six one-dimensional Riemann problems shown here. There was no jump in the x-component of the magnetic field. We set $\gamma=5 / 3$ in all our test problems except test problem number 3 , which had $\gamma=2$.

\begin{tabular}{|l|l|l|l|l|l|l|l|l|l|}
\hline Figure & $\rho$ & $\mathrm{P}$ & $\mathrm{v}_{\mathrm{x}}$ & $\mathrm{v}_{\mathrm{y}}$ & $\mathrm{v}_{\mathrm{z}}$ & $\mathrm{B}_{\mathrm{y}}$ & $\mathrm{B}_{\mathrm{z}}$ & $\mathrm{B}_{\mathrm{x}}$ & $\mathrm{t}_{\text {stop }}$ \\
\hline $1 \mathrm{~L}$ & 1.0 & 1 & 0 & 0 & 0 & 0 & 0 & 1.0 & 0.25 \\
\hline $1 \mathrm{R}$ & 0.1 & 1 & 0 & 0 & 0 & 0 & 0 & & \\
\hline $2 \mathrm{~L}$ & $1 / \sqrt{4 \pi}$ & 1 & -1 & 1 & -1 & -1 & 1 & 1 & 0.25 \\
\hline $2 \mathrm{R}$ & $1 / \sqrt{4 \pi}$ & 1 & -1 & -1 & -1 & 1 & 1 & & \\
\hline $3 \mathrm{~L}$ & 1 & 1 & 0 & 0 & 0 & $\sqrt{4 \pi}$ & 0 & $0.75 \sqrt{4 \pi}$ & 0.1 \\
\hline $3 \mathrm{R}$ & 0.125 & 0.1 & 0 & 0 & 0 & $-\sqrt{4 \pi}$ & 0 & & \\
\hline $4 \mathrm{~L}$ & 1 & 1 & 0 & 0 & 0 & $\sqrt{4 \pi}$ & 0 & $\sqrt{4 \pi}$ & 0.15 \\
\hline $4 \mathrm{R}$ & 0.2 & 0.1 & 0 & 0 & 0 & 0 & 0 & & \\
\hline $5 \mathrm{~L}$ & 1.08 & 0.95 & 1.2 & 0.01 & 0.5 & 3.6 & 2 & 2 & 0.2 \\
\hline $5 \mathrm{R}$ & 1 & 1 & 0 & 0 & 0 & 4 & 2 & & \\
\hline $6 \mathrm{~L}$ & 0.15 & 0.28 & 21.55 & 1 & 1 & -2 & 1 & 0 & 0.04 \\
\hline $6 \mathrm{R}$ & 0.1 & 0.1 & -26.45 & 0 & 0 & 2 & 1 & & \\
\hline
\end{tabular}

We have successfully run all the one-dimensional MHD Riemann test problems from Ryu and Jones [50] and Dai and Woodward [22]. In total, that would result in about seventeen test problems. It is certainly excessive to show the results from so many test problems. For that reason, we first run two test problems that show the FVS Riemann solver's ability to handle isolated contact discontinuities and Alfven waves. Then we show four representative test problems that are drawn from the above-mentioned references. For all the results we used a uniform one-dimensional mesh with 400 zones over the domain $[-0.5,0.5]$. The discontinuity was set up in the middle of the domain. The final stopping time is given in Table I. In all the figures we show the variables from the FVS-based Riemann solver with crosses. The solid curve shows the same variable obtained from a linearized Riemann solver for MHD and provides a point of comparison. In the paragraphs below we discuss the test problems and their import. 


\section{IV.1) Isolated, Stationary Contact Discontinuity}

In this problem we catalogue the performance of our Riemann solver in preserving isolated, stationary contact discontinuities on the mesh. Any good Riemann solver should have this very desirable property. Fig. 1 shows the density profile from test problem 1 . Only the density is of interest, and so, only the density variable is shown. We see that the density profile is preserved very sharply on the mesh without any intermediate points. This is a natural consequence of the flux vector splitting and the fact that we include intermediate waves in our HLLEM Riemann solver for the pressure sub-system. It is also worth pointing out that if the HLL Riemann solver had been used for the pressure sub-system, the contact discontinuity would have been smeared significantly by the time this problem reaches its final stopping time. The inclusion of the HLLEM anti-diffusive correction in eqns. (3.26) and (3.27) was crucial for capturing isolated, stationary contact discontinuities in MHD. This test problem, therefore, illustrates the value of using the HLLEM Riemann solver in treating the pressure sub-system.

We mention in passing that the linearized Riemann solver from Balsara [2], the HLLD Riemann solver of Miyoshi and Kusano [42] and the HLLEM Riemann solver from Dumbser and Balsara [24] can also successfully preserve stationary contact discontinuities on the mesh without further smearing. HLLC Riemann solvers by Gurski [31] and Li [36] should also have this beneficial property.

\section{IV.2) Isolated, Stationary Alfven Wave Discontinuity}

In this problem we catalogue the performance of our Riemann solver in preserving isolated, stationary Alfven wave discontinuities on the mesh. Figs. 2a and 2b show the y-velocity and the y-component of the magnetic field. Test problem 2 is designed so that these two variables carry the discontinuity in the Alfven wave. We see that the Alfven wave discontinuity is indeed smeared. Indeed, we ran this problem twice, using the HLLEM Riemann solver for the

pressure sub-system and the HLL Riemann solver for the pressure sub-system. We found that both Riemann solvers produce an equal amount of smearing. This highlights the fact that setting up a stationary Alfven wave necessarily requires that the flow should be going in one direction while the Alfvenic discontinuity propagates in the opposite direction. This explains why the FVS-based Riemann solvers are not adept at capturing stationary Alfven wave discontinuities. 
The flux vector splitting, by sheer necessity, has to split the entire system into an advected subsystem and a pressure sub-system. Neither of these sub-systems has an exact eigenvector corresponding to Alfven waves. Consequently, isolated, stationary Alfven wave discontinuities cannot be sharply captured on a computational domain when the FVS-based Riemann solver is used for the MHD system.

For the sake of completeness it is worth mentioning that the linearized Riemann solver from Balsara [2], the HLLD Riemann solver of Miyoshi and Kusano [42] and the HLLEM Riemann solver from Dumbser and Balsara [24] can indeed successfully preserve stationary Alfven wave discontinuities on the mesh without further smearing. This is intimately connected to the fact that they project the entire MHD system into its constituent waves without resort to a flux vector splitting.

\section{IV.3) Brio-Wu Test Problem}

This Riemann problem derives from Brio and $\mathrm{Wu}$ [18] and illustrates the formation of a left-going slow compound wave. The compound wave is most prominently shown by the cusp in the density profile. Figs. 3a, 3b, 3c, 3d and 3e show the density, pressure, x-velocity, y-velocity and $y$-magnetic field for the Brio and Wu test problem. We see that the contact discontinuity in the density variable is indeed crisply preserved, though it is not as sharp as the result of a linearized Riemann solver or the Riemann solver from Dumbser and Balsara [24]. This shows the deficiency of the FVS approach. Even so, all the variables are properly captured.

\section{IV.4) Riemann Problem with Switch-On Fast Shock}

Notice that the transverse magnetic field in this test problem is zero to the right of the initial discontinuity while it is non-zero to the left of the initial discontinuity. Along with the chosen values for the other variables, this conspires to produce a switch-on fast shock. I.e., in this problem we have a right-going fast shock where the transverse magnetic field is zero in front of the shock and non-zero behind it. In other words, the fast shock causes the transverse magnetic field to switch-on in the post-shock region. This phenomenon is purely a consequence of MHD being a non-convex system. Figs. 4a, 4b, 4c, 4d and 4e show the density, pressure, $\mathrm{x}-$ velocity, y-velocity and y-magnetic field for the Riemann problem with a switch-on fast shock. Fig. 4e clearly shows that the transverse magnetic field switches on in the post-shock region. Fig. 
4 shows that this problem is correctly simulated by the FVS-based Riemann solver for MHD. It, therefore, makes the point that the Riemann solver designed here is sufficiently capable when it comes to capturing features that derive from the non-convexity of the MHD equations.

\section{IV.5) Riemann Problem with all Seven Waves}

In general, the MHD Riemann problem can produce a system with up to seven distinct waves. Test problem 5 shows such a Riemann problem. Figs. 5a, 5b, 5c, 5d, 5e, 5f and 5g show the density, pressure, $\mathrm{x}$-velocity, y-velocity, z-velocity, and y- and z-magnetic fields for test problem number 5 which has all seven waves that can develop in the MHD system. We see that this problem produces a pair of fast and slow right-going magnetosonic shocks and a pair of fast and slow left-going magnetosonic shocks. The shocks reveal themselves most naturally in the pressure variable in Fig. 5b. In between each pair of fast and slow magnetosonic shocks we have a rotational discontinuity. The rotational discontinuities are most clearly visible in Figs. 5d and 5f for the transverse velocity and the transverse magnetic field. The density variable in Fig. 5a also shows the presence of a contact discontinuity. We see that our FVS-based Riemann solver has captured all these different families of discontinuities properly.

\section{IV.6) MHD Riemann Problem that is the Analogue of the Noh Problem}

Test problem number 6 is the analogue of the Noh problem. It consists of two colliding streams of magnetized plasma that are colliding with one another at very high speed. The problem gives rise to two very strong fast shocks which propagate to either side of a central contact discontinuity. Figs. 6a, 6b, 6c, 6d, 6e, 6f and 6g show the density, pressure, x-velocity, yvelocity, z-velocity, and y- and z-magnetic fields for test problem number 6 which is the MHD analogue of the Noh problem. The strong shocks can be identified most easily in the pressure variable in Fig. 6b. The contact discontinuity is most easily seen via the density variable in Fig. 6a. We see that the two strong shocks are free of post-shock oscillations. The contact discontinuity propagates very slowly with respect to the mesh and is very crisply captured. It does have a small undershoot, but the solution from the linearized Riemann problem also shows the same slight deficiency. Overall, we see that the FVS-based Riemann solver successfully captures all the features in this very stringent test problem.

\section{V) Conclusions}


The goal of this paper was to explore flux vector splittings for the MHD system. Following the philosophy of Toro and Vázquez [55] we have explored three possible flux vector splittings of the MHD equations. The first one had obvious deficiencies with respect to hyperbolicity. The second one could possibly be serviceable in situations where the gas pressure dominates over magnetic pressure. The third one is the most promising because it retains a nice hyperbolic structure in all limits, including situations where the gas pressure does not dominate magnetic pressure. This third, favorable flux vector splitting was then studied in much greater detail.

The pressure sub-system is crucial to finding a successful flux vector splitting. The eigenstructure of the successful flux vector splitting for MHD is thoroughly explored and orthonormalized left and right eigenvectors are explicitly catalogued. We present a very efficient solution of the Riemann problem formed by the pressure sub-system for the MHD equations. Our method consists of applying a newly-discovered HLLEM Riemann solver by Dumbser and Balsara [24] to the pressure sub-system. This new HLLEM Riemann solver is very efficient because it only requires the evaluation of the eigenvectors of the intermediate waves that are of interest. For MHD, these waves include the Alfven waves and the entropy wave. Once the pressure sub-system is solved, the advection sub-system follows naturally. The method presented here also works very well for Euler flow when the intermediate waves are taken to be the entropy wave and the shear waves.

The main advantages of our flux vector splitting for MHD are its simplicity and its efficiency. Downstream, it might also show its utility in enabling the construction of semiimplicit schemes for MHD. Our FVS successfully captures isolated, stationary contact discontinuities in MHD. However, we explain why any FVS for MHD is not proficient at capturing isolated, stationary Alfvenic discontinuities. Several stringent one-dimensional Riemann problems are presented to show that the method works successfully.

In this paper we have focused on one-dimensional tests. In a separate publication we will show that this FVS-based Riemann solver is easily extended so as to yield a truly multidimensional Riemann solver. Multidimensional tests will, therefore, be presented in a subsequent publication. 


\section{Acknowledgements}

DSB acknowledges support via NSF grants NSF-ACI-1307369, NSF-DMS-1361197 and NSF-ACI-1533850. DSB also acknowledges support via NASA grant NASA-NNX 12A088G. Several simulations were performed on a cluster at UND that is run by the Center for Research Computing. Computer support on NSF's XSEDE computing resources is also acknowledged.

\section{References}

[1] R. K. Agarwal, J. Augustinus, D. W. Halt, A Comparative Study of Advection Upwind Split (AUSM) and Wave/Particle Split (WPS) Schemes for Fluid and MHD Flows, in: AIAA 30th Plasmadynamics and Lasers Conference. Norfolk, VA: AIAA Paper, pp. 99-3613

[2] D.S. Balsara, Linearized formulation of the Riemann problem for adiabatic and isothermal magnetohydrodynamics, Astrophysical Journal Supplement 116 (1998) 119

[3] D.S. Balsara, Divergence-free adaptive mesh refinement for magnetohydrodynamics, Journal of Computational Physics 174 (2001) 614-648

[4] D. S. Balsara, Second-order-accurate schemes for magnetohydrodynamics with divergencefree reconstruction, Astrophysical Journal Supplement 151 (2004) 149-184

[5] D.S. Balsara, Divergence-free reconstruction of magnetic fields and WENO schemes for magnetohydrodynamics, J. Comput. Phys., 228 (2009) 5040-5056

[6] D.S. Balsara, Multidimensional HLLE Riemann solver; Application to Euler and Magnetohydrodynamic Flows, J. Comput. Phys., 229 (2010) 1970-1993

[7] D.S. Balsara, A two-dimensional HLLC Riemann solver for conservation laws: Application to Euler and magnetohydrodynamic flows, Journal of Computational Physics 231 (2012) 74767503

[8] Balsara, D.S., Self-Adjusting, Positivity Preserving High Order Schemes for Hydrodynamics and Magnetohydrodynamics, Journal of Computational Physics, 231 (2012b) 7504-7517 
[9] D.S. Balsara, M. Dumbser and R. Abgrall, Multidimensional HLL and HLLC Riemann Solvers for Unstructured Meshes - With Application to Euler and MHD Flows, Journal of Computational Physics 261 (2014) 172-208

[10] D.S. Balsara, Multidimensional Riemann Problem with Self-Similar Internal Structure Part I - Application to Hyperbolic Conservation Laws on Structured Meshes, Journal of Computational Physics 277 (2014) 163-200

[11] D.S. Balsara and M. Dumbser, Multidimensional Riemann Problem with Self-Similar Internal Structure - Part II - Application to Hyperbolic Conservation Laws on Unstructured Meshes, to appear, Journal of Computational Physics (2015)

[12] D.S. Balsara, Three Dimensional HLL Riemann Solver for Structured Meshes; Application to Euler and MHD Flow, Journal of Computational Physics 295 (2015) 1-23

[13] D.S. Balsara and M. Dumbser, Divergence-Free MHD on Unstructured Meshes using High Order Finite Volume Schemes Based on Multidimensional Riemann Solvers, Journal of Computational Physics 299 (2015b) 687-715

[14] D.S. Balsara, J. Vides, K. Gurski, B. Nkonga, M. Dumbser, S. Garain, E. Audit, A TwoDimensional Riemann Solver with Self-Similar Sub-Structure - Alternative Formulation Based on Least Squares Projection, accepted, Journal of Computational Physics (2015)

[15] D.S. Balsara , B. Nkonga, M. Dumbser and C.-D. Munz, Formulating Multidimensional Riemann Solvers in Similarity Variables - Part III:A Multidimensional Analogue of the HLLEM Riemann Solver for Conservative Hyperbolic Systems, submitted, Journal of Computational Physics (2016)

[16] P. Batten, N. Clarke, C. Lambert and D.M. Causon, On the choice of wavespeeds for the HLLC Riemann solver, SIAM J. Sci. Comput., 18, (1997) 1553-1570

[17] S.J. Billett and E.F. Toro, On WAF-type schemes for multidimensional hyperbolic conservation laws, Journal of Computational Physics, 130 (1997) 1-24

[18] M.Brio, and C.C. Wu, An upwind differencing scheme for the equations of ideal magnetohydrodynamics, Journal of Computational Physics, 75 (1988) 400-422

[19] P. Cargo \& G. Gallice, Roe matrices for ideal MHD and systematic construction of Roe matrices for systems of conservation laws, J. Comput. Phys., 136 (1997) 446

[20] A. Chakraborty and E.F. Toro. Development of an approximate Riemann solver for the steady supersonic Euler equations. The Aeronautical Journal. Vol. 98, pages 325-339, 1994. 
[21] P. Colella, A direct Eulerian MUSCL scheme for gas dynamics, SIAM, J. Sci. Statist. Comput., 6 (1985) 104

[22] Dai, W. and Woodward, P.R., Extension of the piecewise parabolic method to multidimensional ideal magnetohydrodynamics, Journal of Computational Physics, 115 (1994) 485-514

[23] M. Dumbser and E. F. Toro. A simple extension of the Osher Riemann solver to nonconservative hyperbolic systems, Journal of Scientific Computing, 48:70 (2011) 88

[24] M. Dumbser and D.S. Balsara, A New, Efficient Formulation of the HLLEM Riemann Solver for General Conservative and Non-Conservative Hyperbolic Systems, accepted, Journal of Computational Physics (2015)

[25] M. Dumbser and V. Casulli, A conservative, weakly nonlinear semi-implicit finite volume scheme for the compressible Navier-Stokes equations with general equation of state, Applied Mathematics and Computation 000 (2015) 1-19

[26] B.Einfeldt, On Godunov-type methods for gas dynamics, SIAM J. Numer. Anal., 25(3) (1988) 294-318

[27] B.Einfeldt, C.-D. Munz, P.L. Roe \& B. Sjogreen, On Godunov-type methods near low densities, J. Comput. Phys., 92 (1991) 273-295

[28] S.A.E.G. Falle, On the inadmissibility of non-evolutionary shocks, Journal of Plasma Physics, 65 (2001) 29-58

[29] P. Glaister, An approximate linearised Riemann solver for the three-dimensional euler equations for real gases using operator splitting, 77 (1988) 361-383

[30] S.K. Godunov, Finite Difference Methods for the Computation of Discontinuous Solutions of the Equations of Fluid Dynamics, Mathematics of the USSR, Sbornik. 47 (1959) 271-306

[31] K.F. Gurski, An HLLC-type approximate Riemann solver for ideal magnetohydrodynamics, SIAM J. Sci. Comput. 25 (2004) 2165

[32] S.-H. Han, J.-I. Lee, K. H. Kim, Accurate and robust pressure weight advection upstream splitting method for magnetohydrodynamics equations, AIAA journal 47 (2009) 970-981

[33] A. Harten, High resolution schemes for conservation laws, Journal of Computational Physics, 49 (1983) 357-393 
[34] A. Harten, P.D. Lax and B. van Leer, On upstream differencing and Godunov-type schemes for hyperbolic conservation laws, SIAM Rev. (1983) 25, 289-315

[35] Jeffrey, A., \& Taniuti, T. 1964, Nonlinear Wave Propagation (New York: Academic)

[36] S.-T. Li, An HLLC Riemann solver for magnetohydrodynamics, J. Comput. Phys., 203 (2005) 344

[37] Liou M.S., A sequel to AUSM: AUSM+, J. Comput. Phys., (1996) 129:364-82

[38] Liou M.S., Recent progress and applications of AUSM+. In: Sixteenth International Conference on Numerical Methods in Fluid Dynamics. Lecture Notes in Physics, vol. 515. Springer-Verlag; (1998) p. 302-7.

[39] Liou M.S., A Sequel to AUSM, Part II: AUSM+ for all Speeds, J. Comput. Phys. (2006) 214:137-70.

[40] Liou M.S., Steffen C.J., A new flux splitting scheme, J. Comput. Phys., (1993) 107:23-39.

[41] Liou M.S., van Leer B., Shuen J.S., Splitting of inviscid fluxes for real gases, J. Comput. Phys. (1990) 87:1-24.

[42] T. Miyoshi and K. Kusano, A multi-state HLL approximate Riemann solver for ideal magnetohydrodynamics, J. Comput. Phys., 208 (2005) 315-344

[43] S. Noelle, G. Bispen, K.R. Arun, M. Lukacova-Medvidova and C.D. Munz, An asymptotic preserving all Mach number scheme for the Euler equations of gas dynamics, (2014)

[44] S. Osher and F. Solomon, Upwind Difference Schemes for Hyperbolic Systems of Conservation Laws, Mathematics of Computation, 38(158) (1982) 339

[45] J. H. Park and C.-D. Munz, Multiple pressure variables methods for fluid flow at all Mach numbers, Internat. J. Numer. Methods Fluids, 49(8) (2005) 905-931

[46] P.L. Roe, Approximate Riemann solver, parameter vectors and difference schemes, Journal of Computational Physics 43 (1981) 357-372

[47] P.L. Roe and J. Pike, Efficient Construction and Utilisation of Approximate Riemann Solutions in Computing Methodr in Applied Science and Engineering VI, edited by R. Glowinski and J.-L. Lions (North-Holland, Amsterdam, 1984), p. 499 
[48] P. L. Roe and D. S. Balsara, Notes on the eigensystem of magnetohydrodynamics, SIAM Journal of applied Mathematics 56 (1996), 57

[49] V.V. Rusanov, Calculation of interaction of non-steady shock waves with obstacles, J. Comput. Math. Phys. USSR, 1 (1961) 267

[50] Ryu, D. and Jones,T.W., Numerical MHD in astrophysics: algorithm and tests for onedimensional flow, Astrophysical Journal, 442 (1995) 228

[51] Y. Shen, G. Zha, M. A. Huerta, E-CUSP scheme for the equations of ideal magnetohydrodynamics with high order WENO scheme, Journal of Computational Physics 231 (2012) 6233-6247

[52] C.-W. Shu and S. J. Osher, Efficient implementation of essentially non-oscillatory shock capturing schemes, Journal of Computational Physics 77 (1988) 439-471

[53] E F Toro, M Spruce and W Speares. Restoration of the contact surface in the Harten-Laxvan Leer Riemann solver. Shock Waves. Vol. 4 (1994) pages 25-34

[54] E F Toro, M Spruce and W Speares, Restoration of the contact surface in the HLL Riemann solver, Technical report CoA 9204. Department of Aerospace Science, College of Aeronautics, Cranfield Institute of Technology. UK. June, 1992

[55] E.F. Toro and Vázquez-Cendón, Flux splitting schemes for the Euler equations, Computers \& Fluids, 70 (2012) 1-12

[56] E. F. Toro, C. Castro and B.J. Lee, A novel numerical flux for the $3 D$ Euler equations with general equation of state, Journal of Computational Physics, accepted, 2015

[57] P. Tiam-Kapen and T. Ghislain, A New Flux Splitting Scheme Based on Toro-Vázquez and HLL Schemes or the Euler Equations, Journal of Computational Methods in Physics, Volume 2014, pp, 2014.

[58] B. van Leer, Toward the Ultimate Conservative Difference Scheme. V. A Second-Order Sequel to Godunov's Method, J. Comput. Phys., 32 (1979) 101

[59] J. Vides, B. Nkonga \& E. Audit, A simple two-dimensional extension of the HLL Riemann solver for hyperbolic conservation laws, J. Comput. Phys., 280(1) (2015) 643-675

[60] W. Xie, H. Li, Z. Tian and S. Pan, A low diffusion flux splitting method for inviscid compressible flow, Computers \& Fluids, Volume 112, Pages 83-93, 2nd May, 2015 
[61] C. M. Xisto, J. C. Pascoa, P. J. Oliveira, A pressure-based method with AUSM-type fluxes for MHD flows at arbitrary Mach numbers, International Journal for Numerical Methods in Fluids 72 (2013) 1165-1182

[62] Z. Xu, D.S. Balsara and H. Du, Divergence-Free WENO Reconstruction-Based Finite Volume Scheme for Ideal MHD Equations on Triangular Meshes, accepted, Communications in Computational Physics (2015)

[63] Z.C. Zha and E. Bilgen, Numerical solutions of Euler equations by using a new flux vector splitting scheme, International Journal for Numerical Methods in Fluids, 17 (1993) 115-144 


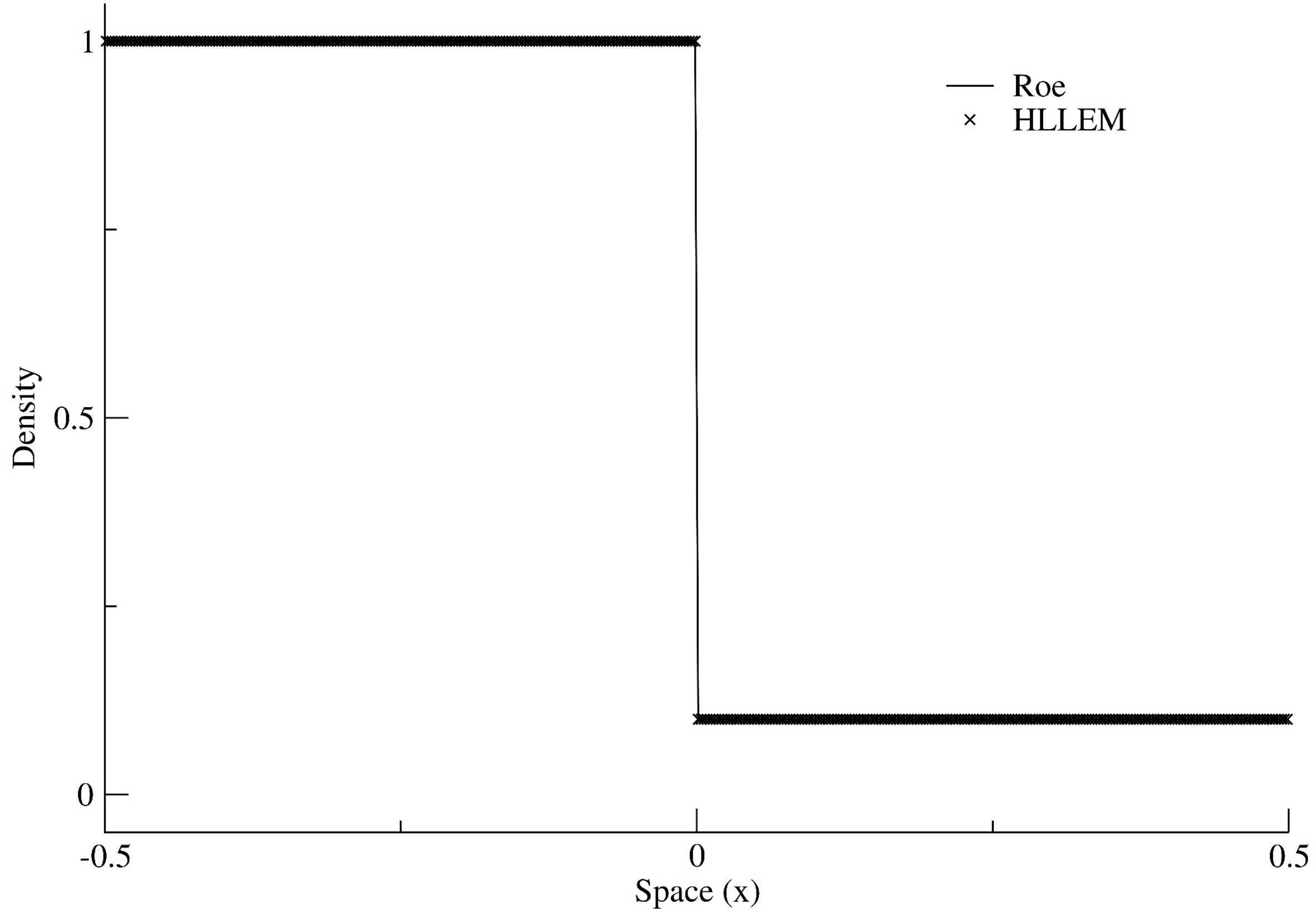

Fig. 1 shows the density profile from test problem 1. Only the density is of interest, and so, only the density variable is shown. The crosses show the results from TV-HLLEM; the solid line shows the results from a linearized Riemann solver for the full MHD system. 
a)

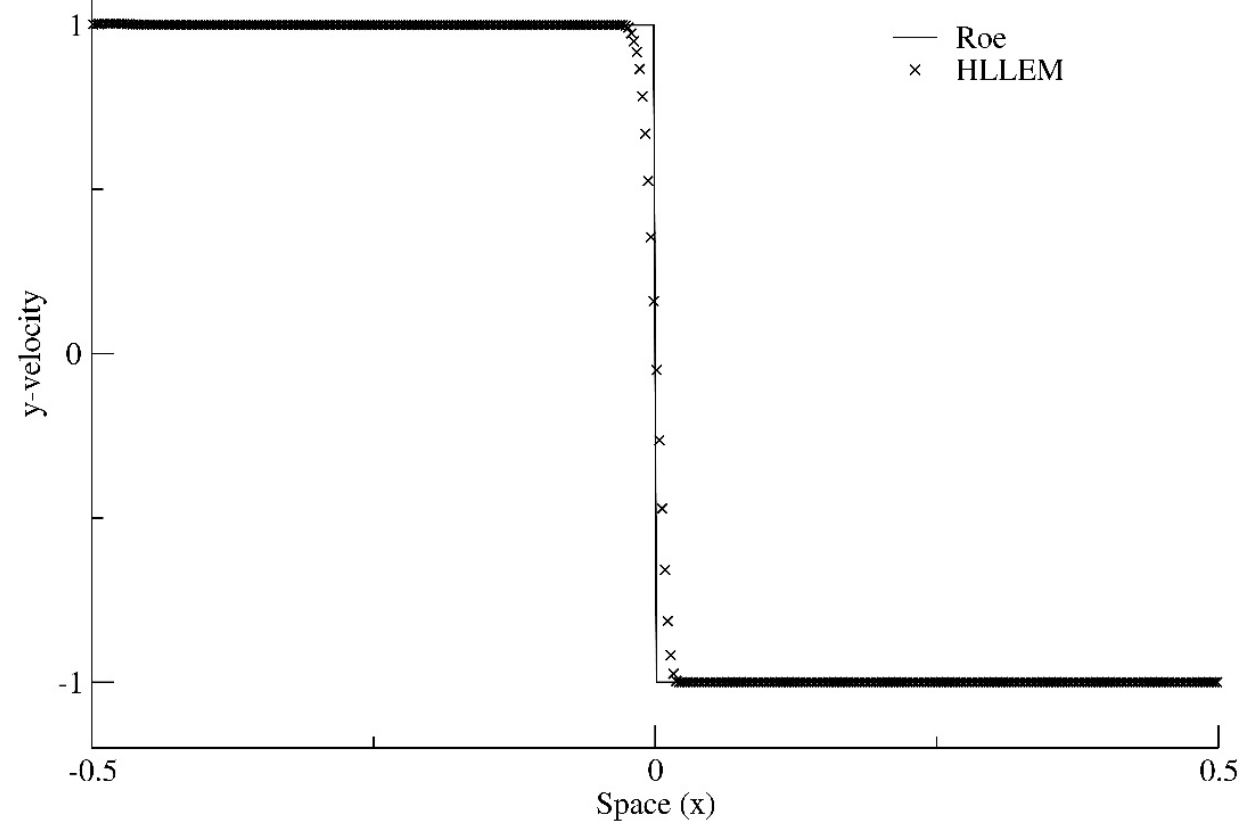

b)

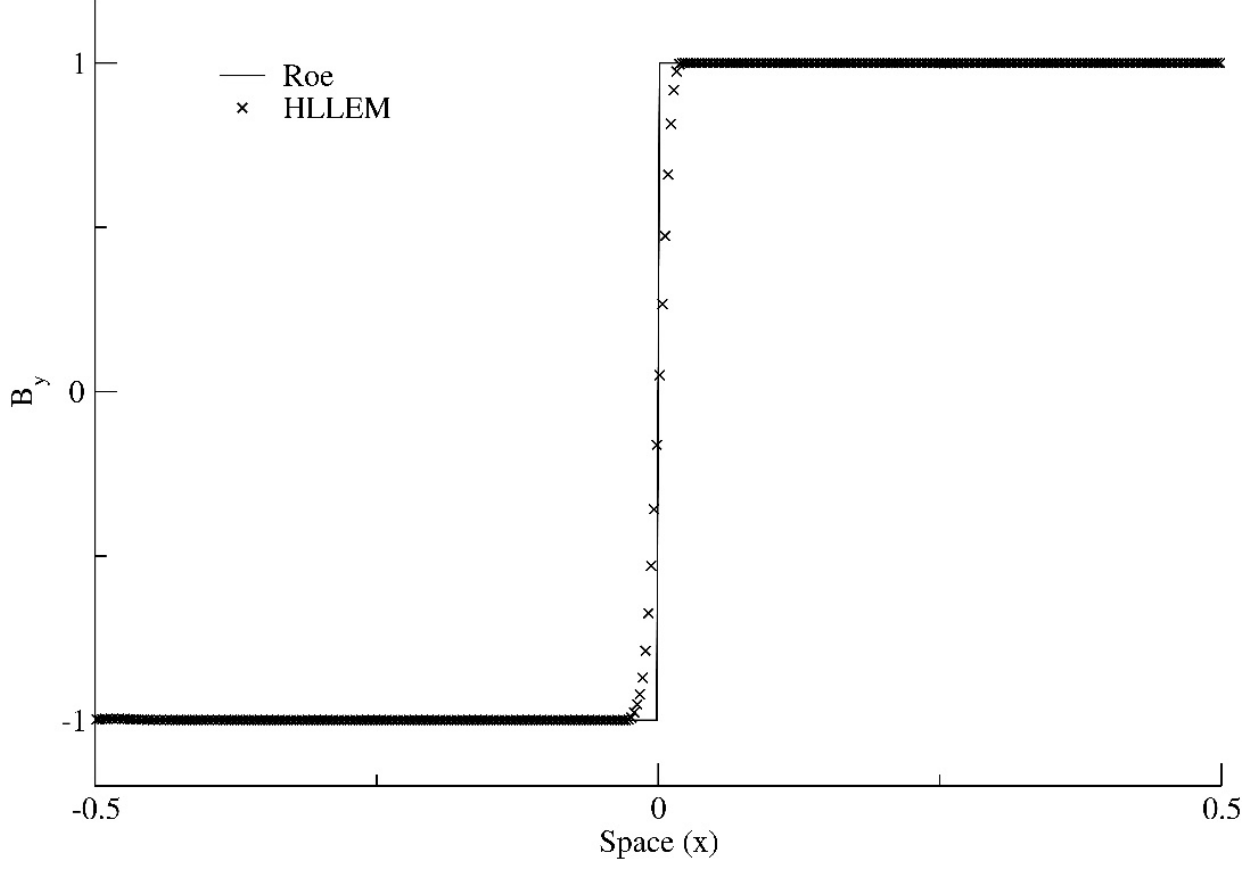

Figs. $2 a$ and $2 b$ show the $y$-velocity and the y-component of the magnetic field. Test problem 2 is designed so that these two variables carry the discontinuity in the Alfven wave. The crosses show the results from TV-HLLEM; the solid line shows the results from a linearized Riemann solver for the full MHD system. 


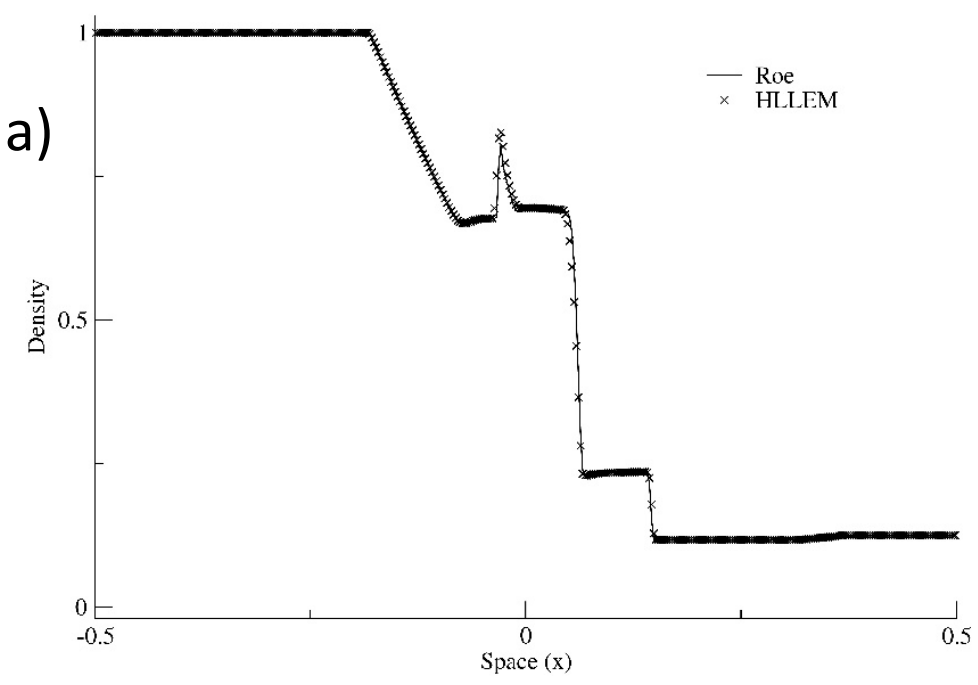

b)

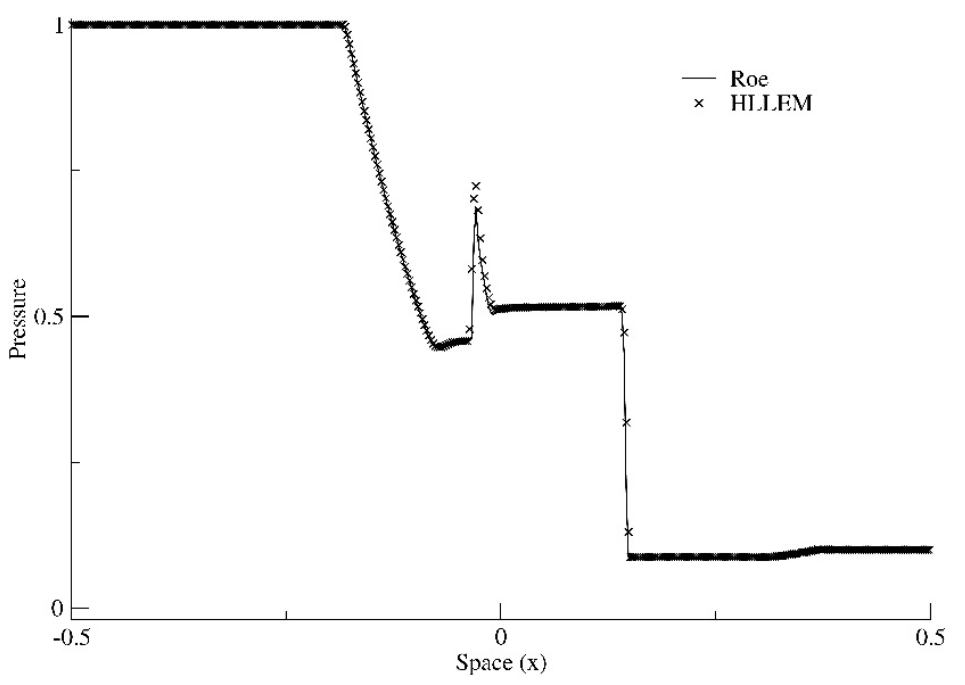

d)

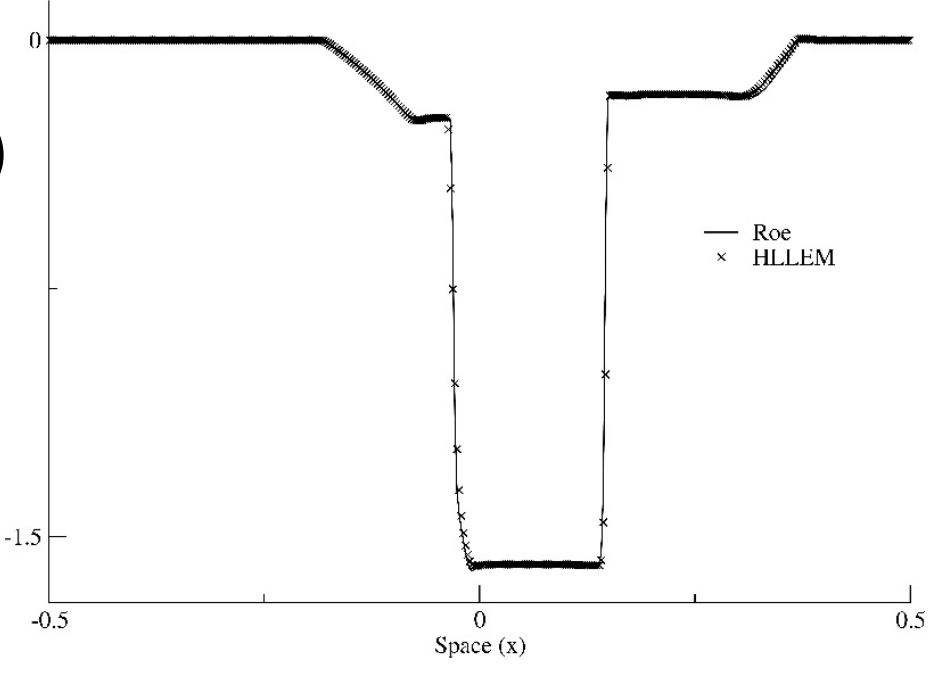

e)

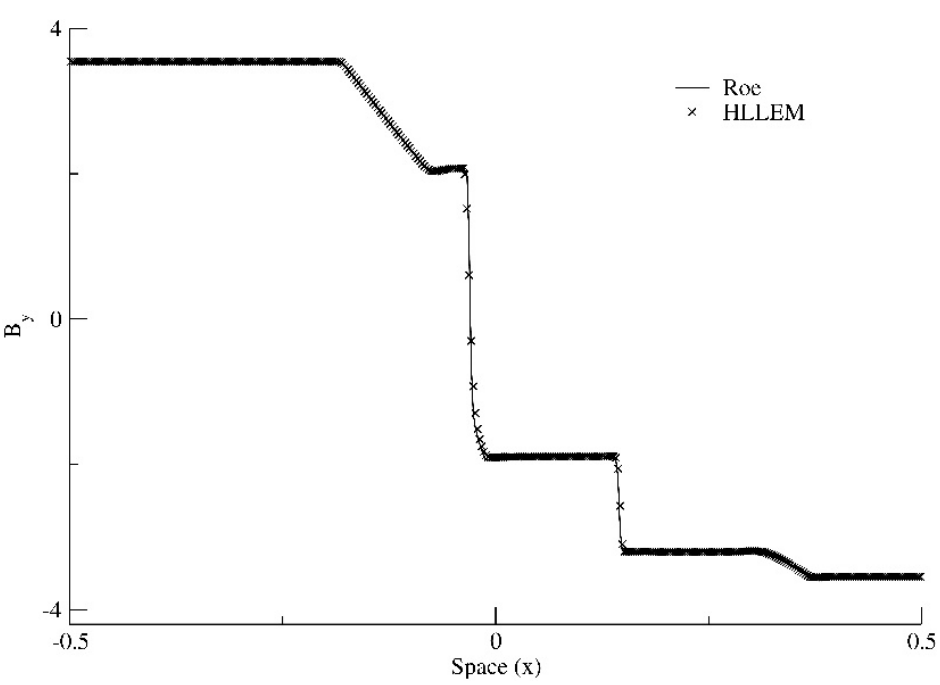

c)

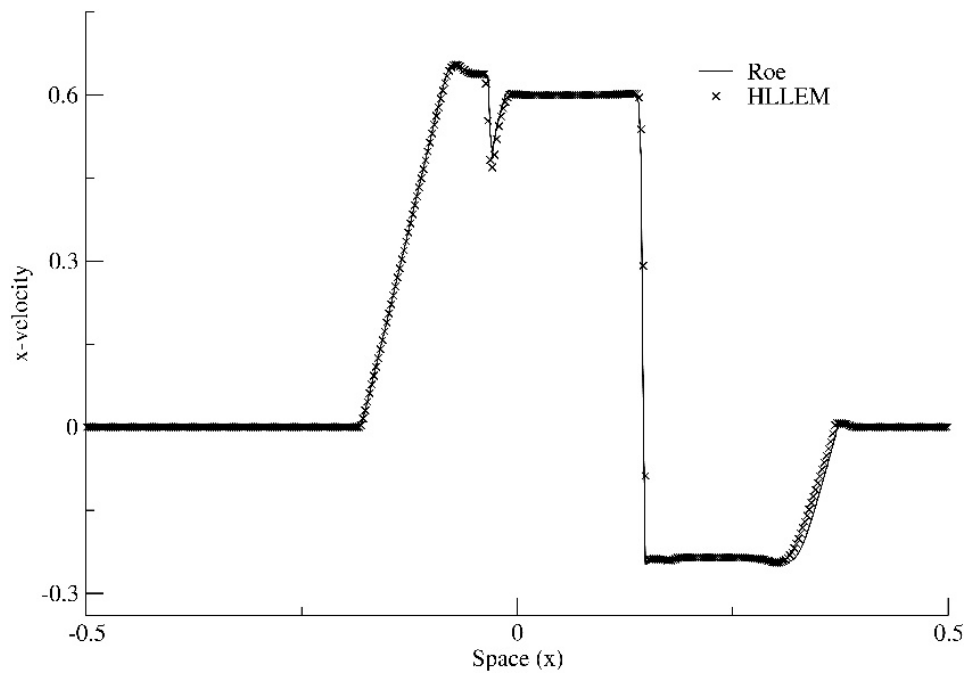

Figs. 3a, 3b, 3c, 3d and 3e show the density, pressure, $x$-velocity, $y$-velocity and $y$-magnetic field for the Brio and Wu test problem. The crosses show the results from TV-HLLEM; the solid line shows the results from a linearized Riemann solver for the full MHD system. 
a)

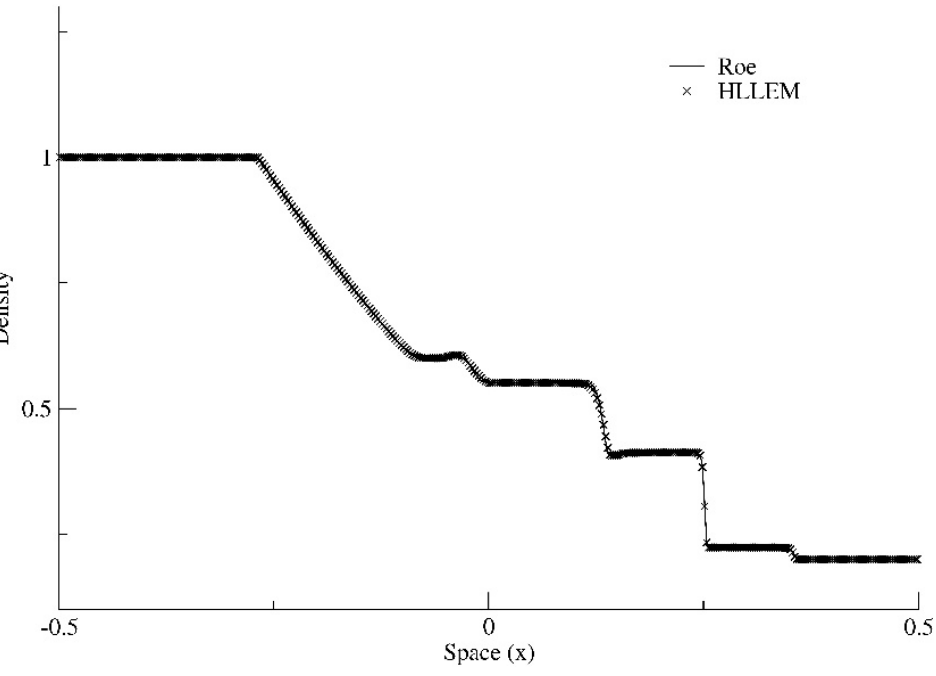

d)

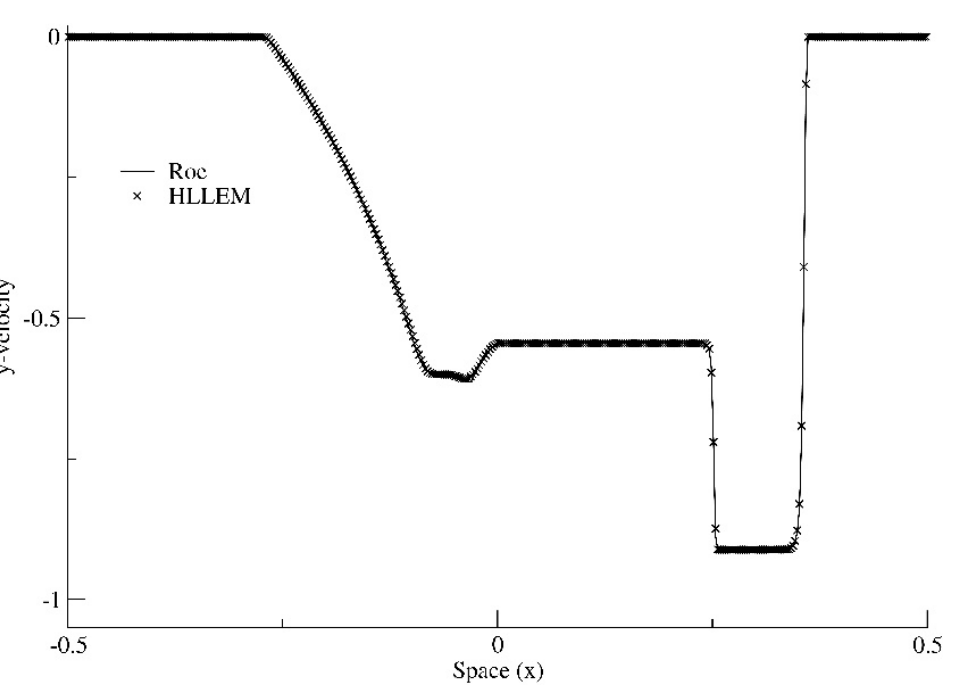

b)

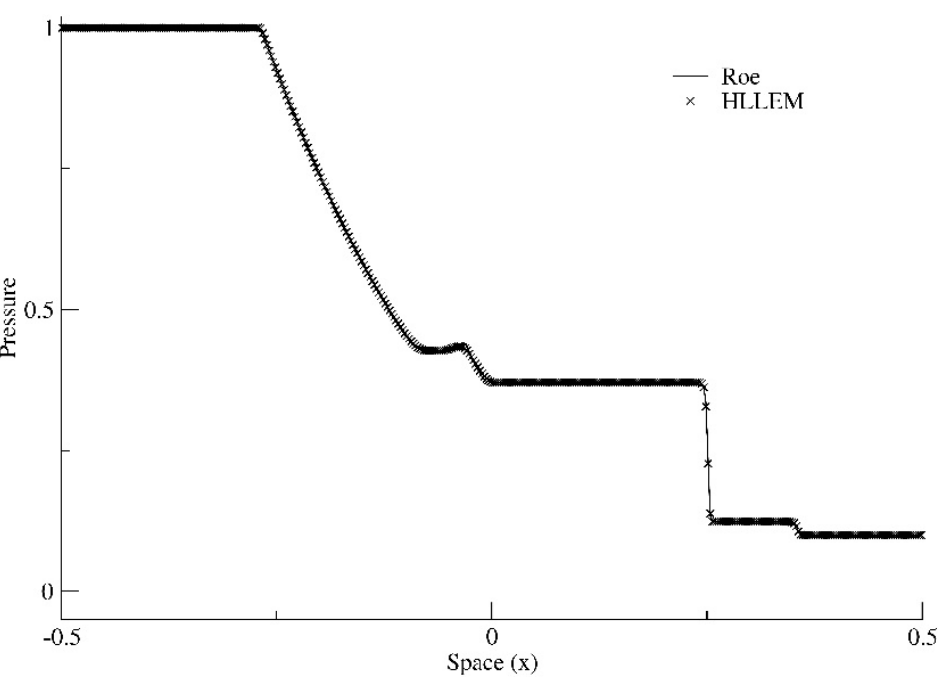

e)

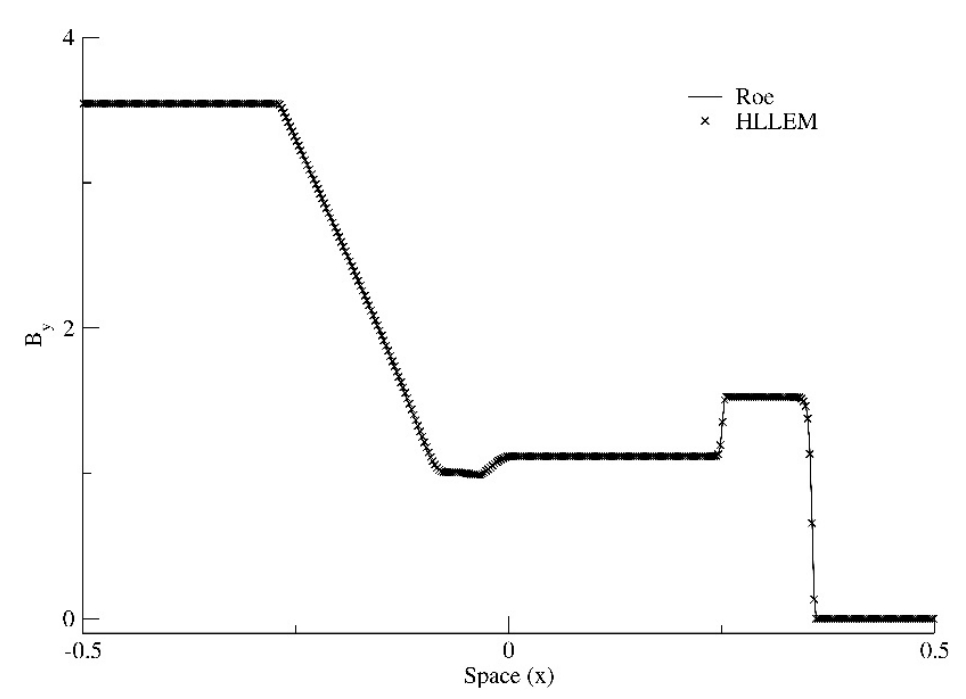

c)

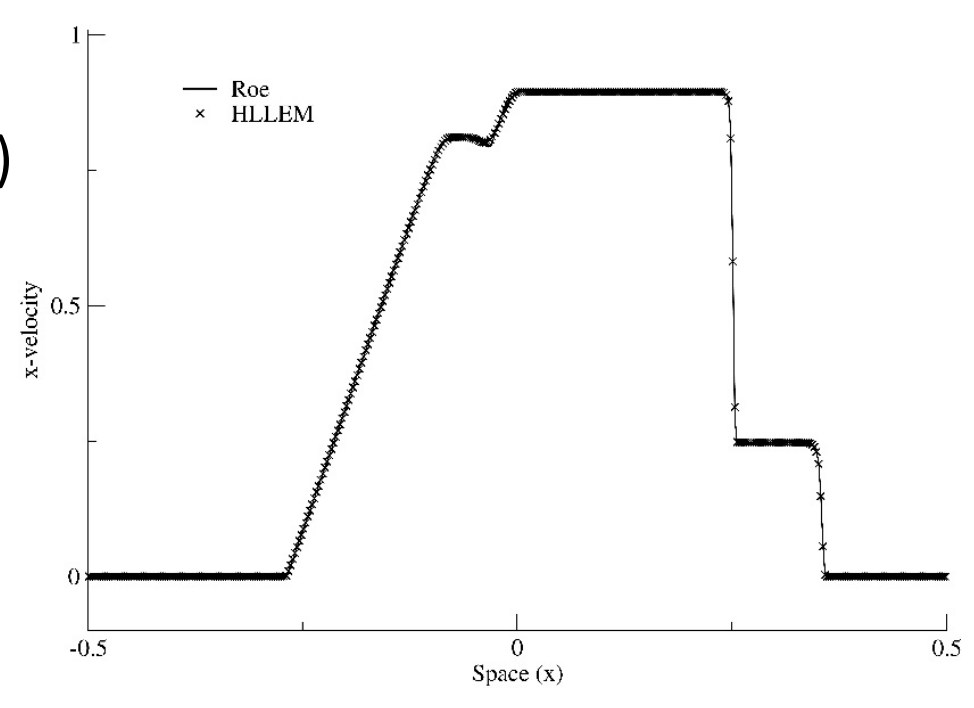

Figs. 4a, 4b, 4c, $4 d$ and $4 e$ show the density, pressure, $x$-velocity, $y$-velocity and $y$-magnetic field for the Riemann problem with a switch-on fast shock. The crosses show the results from TV-HLLEM; the solid line shows the results from a linearized Riemann solver for the full MHD system. 


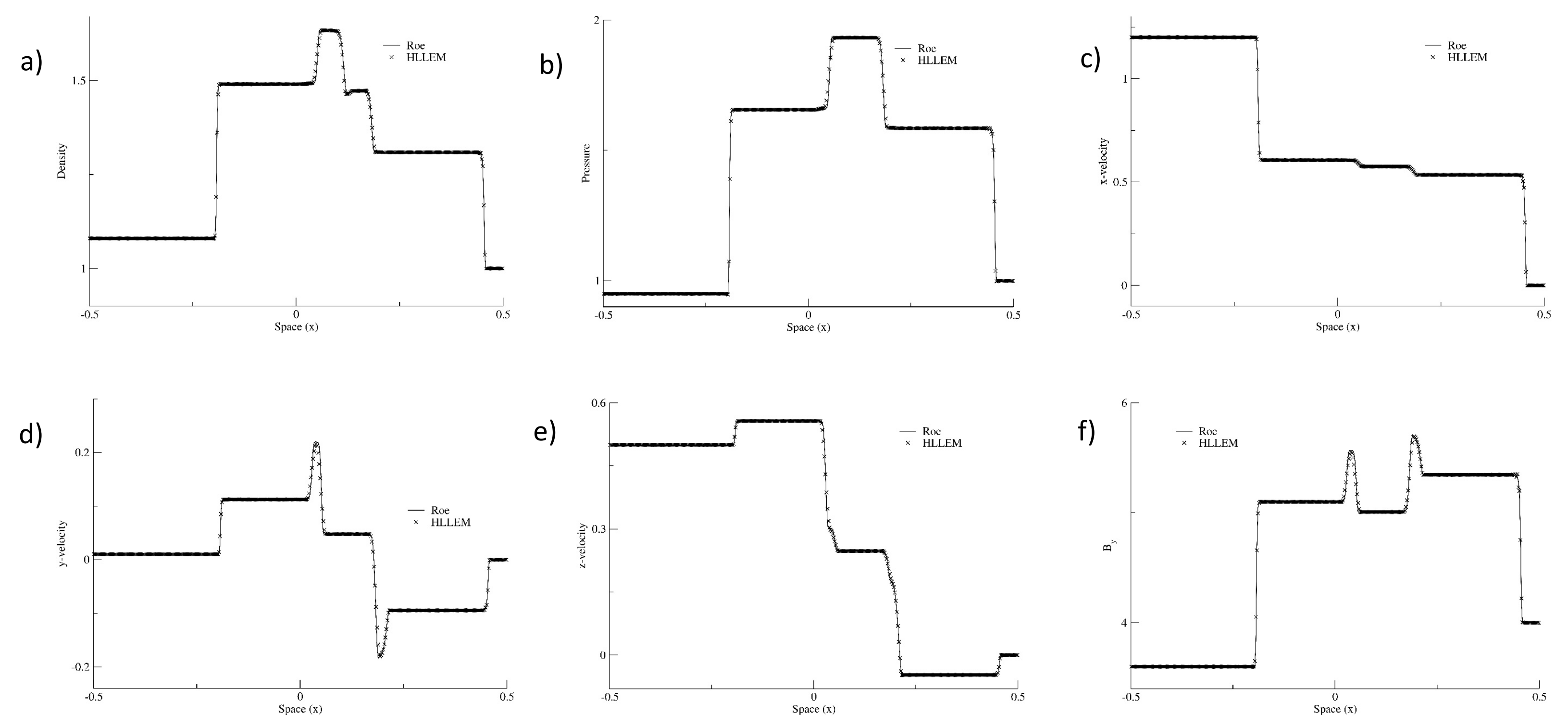


g)

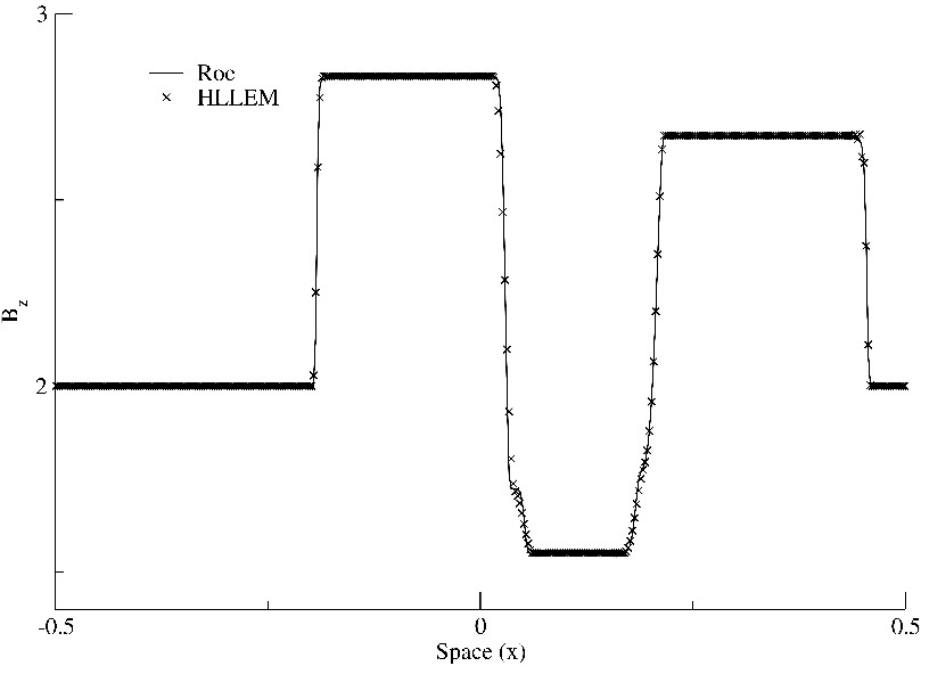

Figs. $5 a, 5 b, 5 c, 5 d, 5 e, 5 f$ and $5 g$ show the density, pressure, $x$-velocity, $y$-velocity, $z$-velocity, and $y$ - and z-magnetic fields for test problem number 5 which has all seven waves that can develop in the MHD system. The crosses show the results from TVHLLEM; the solid line shows the results from a linearized Riemann solver for the full MHD system. 


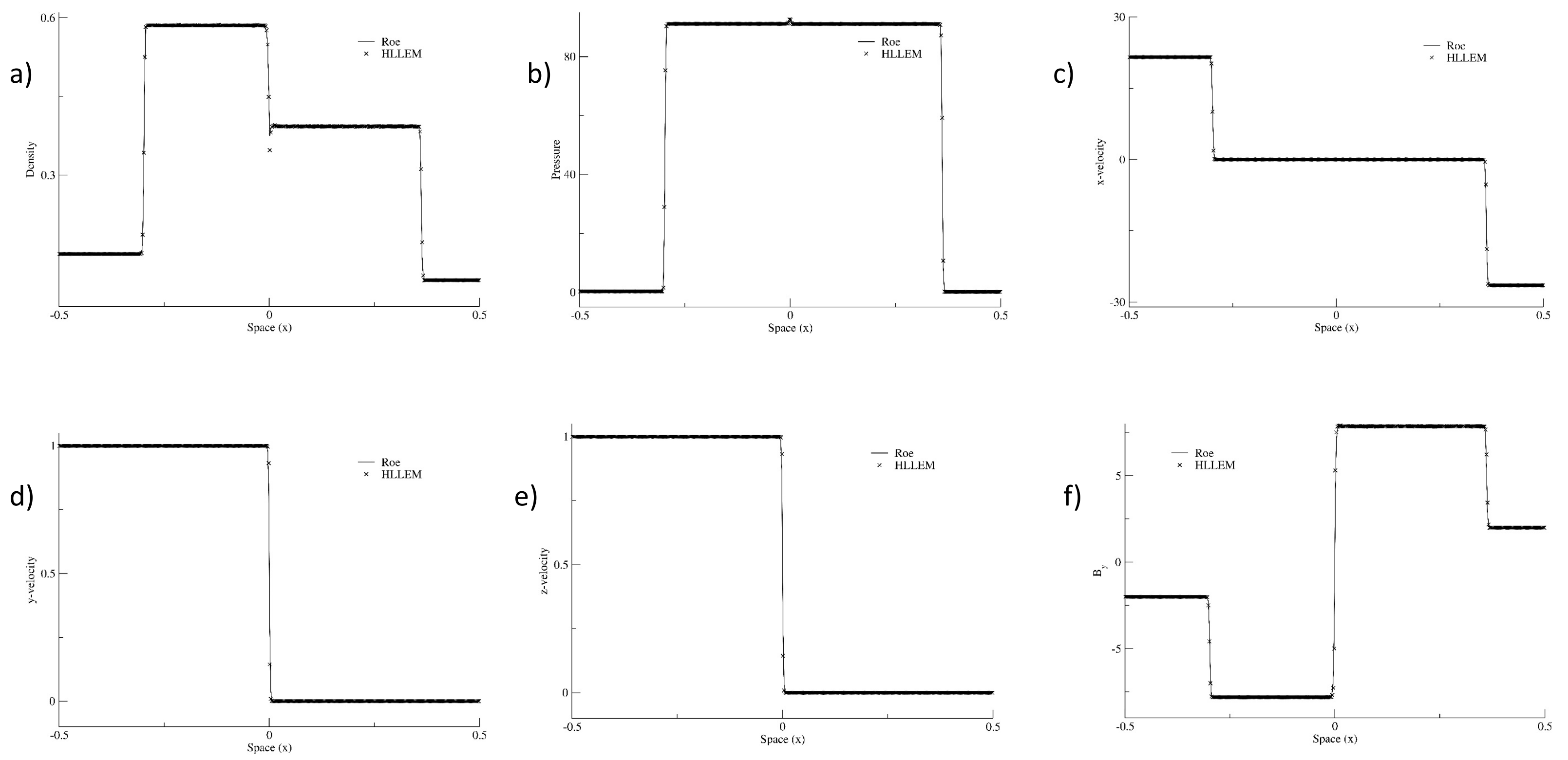


g)

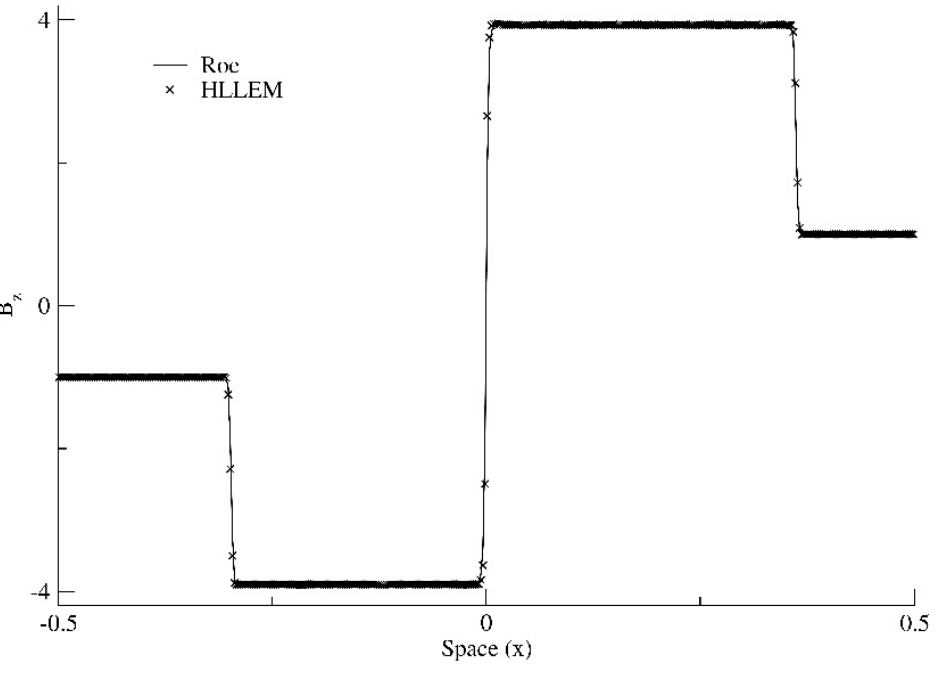

Figs. $6 a, 6 b, 6 c, 6 d, 6 e, 6 f$ and $6 \mathrm{~g}$ show the density, pressure, $x$-velocity, $y$-velocity, z-velocity, and $y$ - and $z$-magnetic fields for test problem number 6 which is the MHD analogue of the Noh problem. The crosses show the results from TV-HLLEM; the solid line shows the results from a linearized Riemann solver for the full MHD system. 